\title{
Inositol 1,4,5-Trisphosphate Receptor and dSTIM Function in Drosophila Insulin-Producing Neurons Regulates Systemic Intracellular Calcium Homeostasis and Flight
}

\author{
Neha Agrawal, ${ }^{\star}$ Gayatri Venkiteswaran, ${ }^{\star}$ Sufia Sadaf, Nisha Padmanabhan, Santanu Banerjee, and Gaiti Hasan \\ National Centre for Biological Sciences, Tata Institute of Fundamental Research, Bangalore 560065, India
}

Calcium $\left(\mathrm{Ca}^{2+}\right)$ signaling is known to regulate the development, maintenance and modulation of activity in neuronal circuits that underlie organismal behavior. In Drosophila, intracellular $\mathrm{Ca}^{2+}$ signaling by the inositol 1,4,5-trisphosphate receptor and the storeoperated channel (dOrai) regulates the formation and function of neuronal circuits that control flight. Here, we show that restoring Ins $\mathrm{P}_{3} \mathrm{R}$ activity in insulin-producing neurons of flightless Ins $\mathrm{P}_{3} \mathrm{R}$ mutants (itpr) during pupal development can rescue systemic flight ability. Expression of the store operated $\mathrm{Ca}^{2+}$ entry (SOCE) regulator dSTIM in insulin-producing neurons also suppresses compromised flight ability of $\mathrm{Ins}_{3} \mathrm{R}$ mutants suggesting that SOCE can compensate for impaired Ins $\mathrm{P}_{3} \mathrm{R}$ function. Despite restricted expression of wild-type InsP ${ }_{3} \mathrm{R}$ and dSTIM in insulin-producing neurons, a global restoration of SOCE and store $\mathrm{Ca}^{2+}$ is observed in primary neuronal cultures from the itpr mutant. These results suggest that restoring Ins $\mathrm{P}_{3} \mathrm{R}$-mediated $\mathrm{Ca}^{2+}$ release and SOCE in a limited subset of neuromodulatory cells can influence systemic behaviors such as flight by regulating intracellular $\mathrm{Ca}^{2+}$ homeostasis in a large population of neurons through a non-cell-autonomous mechanism.

\section{Introduction}

Cellular signals of ionic calcium $\left(\mathrm{Ca}^{2+}\right)$ are generated using an extensive ' $\mathrm{Ca}^{2+}$ toolkit' that consists of calcium channels and pumps on the plasma membrane and the membrane of intracellular stores that help in assembling signaling systems with very different temporal and spatial dynamics (Berridge et al., 2003). Cell surface receptor stimulation activates two closely coupled components of this toolkit; the inositol 1,4,5-trisphosphate receptors $\left(\mathrm{InsP}_{3} \mathrm{R}\right)$ followed by store operated $\mathrm{Ca}^{2+}$ (SOC) channels. Ins $\mathrm{P}_{3} \mathrm{Rs}$ rapidly release $\mathrm{Ca}^{2+}$ from intracellular stores such as the endoplasmic reticulum (ER) while SOC channels function to replenish ER stores from the extracellular milieu and contribute to a longer-term $\mathrm{Ca}^{2+}$ signal. The plasma membrane fourtransmembrane-spanning Orai protein was recently identified as the long-elusive SOC channel (Feske et al., 2006; Vig et al., 2006; Zhang et al., 2006). The exact mechanism of SOC entry (SOCE) is still under investigation but several reports have established that $\mathrm{Ca}^{2+}$ depletion of ER stores is sensed by STIM (stromal interaction molecule), an ER membrane protein, which upon store $\mathrm{Ca}^{2+}$ depletion oligomerizes in the ER, translocates close to the

\footnotetext{
Received July 29, 2009; revised Dec. 2, 2009; accepted Dec. 8, 2009.

This work was supported by core funding from the National Centre for Biological Sciences and a grant from the Department of Biotechnology to G. H. We thank Swarna Mathre for help with pupal dissections, Shalini V. for making the UASdSTIM construct, and Dr. H. Krishnamurthy and the National Centre for Biological Sciences Central ImageFlow Facility for help with imaging.

*N.A. and G.V. contributed equally to this manuscript.

Correspondence should be addressed to: Gaiti Hasan, National Centre for Biological Sciences, Tata Institute of Fundamental Research, Bangalore 560065, India. E-mail: gaiti@ncbs.res.in.

S. Banerjee's present address: Department of Cell and Developmental Biology, University of Pennsylvania, School of Medicine, 1236 BRBII/III Building, 421 Curie Boulevard, Philadelphia, PA 19104.

DOI:10.1523/JNEUROSCI.3668-09.2010

Copyright $\odot 2010$ the authors $\quad 0270-6474 / 10 / 301301-13 \$ 15.00 / 0$
}

plasma membrane and organizes the Orai channel into clusters to bring about SOCE (Liou et al., 2005; Zhang et al., 2005; Cahalan, 2009). Recent findings strongly suggest that Orai and STIM together are necessary and sufficient for SOCE (Hewavitharana et al., 2007 and references therein).

Neuronal $\mathrm{Ca}^{2+}$ signals play an important role in the development, maintenance and modulation of activity in neural circuits (Berridge, 1998; Spitzer, 2002; Borodinsky et al., 2004). The importance of neuronal $\mathrm{InsP}_{3} \mathrm{R}$-mediated $\mathrm{Ca}^{2+}$ signaling for neural circuit function is well recognized (Banerjee and Hasan, 2005; Mikoshiba, 2006). We have previously shown that flight and associated defects observed in itpr gene (which encodes the only Drosophila $\operatorname{Ins}_{3} \mathrm{R}$ ) mutants can be rescued by restoring $\operatorname{Ins} \mathrm{P}_{3} \mathrm{R}$ function in aminergic neurons (Banerjee et al., 2004) and that Ins $\mathrm{P}_{3} \mathrm{R}$-mediated $\mathrm{Ca}^{2+}$ signaling is required at multiple steps for generating the neural circuit responsible for air-puff stimulated Drosophila flight (Banerjee et al., 2006). We also recently identified a requirement for SOCE in Drosophila neurons for regulating flight behavior (Venkiteswaran and Hasan, 2009).

Here, the contribution of intracellular $\mathrm{Ca}^{2+}$ signaling in function and development of the neural circuitry underlying Drosophila flight behavior, has been further investigated. We find that restoring $\operatorname{Ins}_{3} \mathrm{R}$ signaling in the insulin-producing neurons (referred to as DILP2; Drosophila insulin-like peptide 2 neurons) during pupal development can rescue flight and flight physiology defects in itpr mutants. The DILP2 neurons do not overlap with aminergic neurons in pupal and adult brains, though they are closely apposed. Interestingly, expressing wild-type dSTIM in either DILP2 or aminergic neurons can also rescue itpr mutant flight phenotypes suggesting that SOCE can compensate for compromised $\mathrm{InsP}_{3}$ signaling. Moreover, this rescue of systemic itpr 
mutant phenotypes by expression of wild-type $\mathrm{InsP}_{3} \mathrm{R}$ or dSTIM in DILP2 neurons results in a global restoration of neuronal calcium homeostasis. These results suggest that modulation of calcium signaling in a small neuronal subset can influence both cellular and systemic phenotypes.

\section{Materials and Methods}

Drosophila melanogaster strains. itpr ${ }^{\text {ka1091/ug3 }}$ and itpr $r^{\text {wc703/ug3 }}$ are heteroallelic combinations of single point mutants in the itpr gene that were generated in an EMS (ethyl methanesulfonate) screen. Detailed molecular information on these alleles has been published (Joshi et al., 2004). The UASdSTIM ${ }^{+}$transgenic strain on chromosome 2 was generated by injecting embryos using a standard Drosophila embryo microinjection protocol with a pUASTASTIM construct. The construct was generated from a full-length dSTIM cDNA clone (BDGP LD45776). The other strains used are as follows: Dilp2GAL4, provided by Dr. E. Rulifson (Rulifson et al., 2002), embryonic wild-type itpr cDNA (UASitpr ${ }^{+}$) (Venkatesh et al., 2001), GAL80 $0^{t s}$ with two inserts on the second chromosome (generated by Albert Chiang, NCBS, Bangalore, India), $\mathrm{UASdOrai}^{+}$(Venkiteswaran and Hasan, 2009), DdcGAL4 referred in the text as aminergic (Li et al., 2000), a recombinant of Dilp2GAL4 and DdcGAL4 referred to as doubleGAL4 and

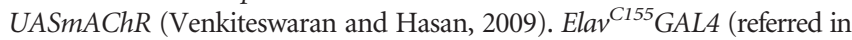
the text as pan-neuronal), UASdicer(III) and UASmCD8GFP(II) were obtained from the Bloomington Stock Centre, Bloomington, IN. UASRNAi strain for dOrai (12221) and dSTIM (47073) (referred in the text as $d s d O r a i$ and $d s d S T I M$, respectively) were obtained from the Vienna Drosophila RNAi Centre, Vienna, Austria (Dietzl et al., 2007), while that for itpr (1063-R2) referred in the text as dsitpr was obtained from the National Institute of Genetics Fly Stocks Centre. The other fly strains used were generated by standard genetic methods using individual mutant and transgenic fly lines described above.

Flight assay. Flight tests were performed as described previously (Banerjee et al., 2004), following minor modifications of the "cylinder drop assay" (Benzer, 1973). Flies were tested in batches of 20 by dropping them into a 1-m-long glass cylinder. Flies that fell through directly into a chilled conical flask kept below were scored as nonfliers (NF), and those that flew and sat on the walls of the cylinder were scored as fliers (Fl). The percentage of fliers was then determined. Computation of mean and SEM was performed on results obtained from at least 100 flies, using Origin 7.5 software (MicroCal). Statistically significant differences between 2 groups were determined by Student's $t$ tests for independent populations. Significant differences were taken at $p<0.05$.

Electrophysiological and video recordings. For electrophysiological and video recordings of responses to an air puff stimulus, flies were anesthetized briefly with diethyl ether or on ice and glued to a thin metal wire between the neck and the thorax with nail polish. To record air puff responses, a gentle mouth-blown air puff stimulus was delivered to the fly kept in a tethered condition. Physiological recordings were performed on the dorsal longitudinal muscles (DLMs) of the giant fiber pathway as described previously (Banerjee et al., 2004). After recovery from anesthesia, an uninsulated tungsten electrode $(0.5 \mu \mathrm{m})$, which had been sharpened by electrolysis, was inserted carefully into the DLM (fiber a) just beneath the cuticle. A similar tungsten electrode was inserted in the abdomen as a reference. Flies were rested for at least 5-10 min after insertion of electrodes before beginning the recording. For measurement of spontaneous activity, flies were kept undisturbed and recordings were performed for $2 \mathrm{~min}$. Air puff-induced recordings were performed for $30 \mathrm{~s}$. All recordings were made using an ISODAM8A (World Precision Instruments) amplifier with filter set up $30 \mathrm{~Hz}$ (low pass) to $>10 \mathrm{kHz}$ (high pass). Gap-free mode of pClamp8 (Molecular Devices) was used to digitize the data $(10 \mathrm{kHz})$ on a Pentium 5 computer equipped with Digidata 1322A (Molecular Devices). Data were analyzed using Clampfit (Molecular Devices) and plotted using Origin 7.5 software (MicroCal). Response to air puff and spontaneous activity were recorded from $\sim 10$ or more flies for every genotype.

Immunohistochemistry. Immunohistochemistry was performed on Drosophila pupal and adult brains, expressing a membrane bound GFP (UASmCD8GFP) with the Dilp2GAL4, after fixing the dissected tissue in
$4 \%$ paraformaldehyde. The following primary antibodies were used: rat anti-Ddc (1:400; provided by Dr. J. Hirsh, University of Virginia, VA), rabbit anti-GFP antibody (1:10,000; Invitrogen) and a monoclonal antiserotonin antibody (NeoMarkers, 1:50; Lab Vision Corp.). Fluorescent secondary antibodies were used at a dilution of 1:400 as follows: antirabbit Alexa Fluor 488 and anti-rat Alexa Fluor 633 (Invitrogen), antimouse Rhodamine Red (Jackson ImmunoResearch Laboratories Inc.). Confocal analysis was performed on a Zeiss LSM 510 Meta microscope (Carl Zeiss) or an Olympus Confocal FV1000 microscope using $20 \times 0.9$ numerical aperture (NA) or $63 \times 1.4 \mathrm{NA}$ objectives. Confocal data were acquired as image stacks of separate channels and combined and visualized as three-dimensional projections using the LSM5 version 3.2/SP2 software (Carl Zeiss) or FV10-ASW 1.3 viewer (Olympus Corporation).

Primary neuronal cultures from Drosophila larvae. Procedures for culturing Drosophila larval neurons have been described (Venkiteswaran and Hasan, 2009). Primary cultures of dissociated Drosophila larval neurons were plated in $200 \mu \mathrm{l}$ of Drosophila M1 medium (30 mM HEPES/150 mм NaCl$/ 5$ mм KCl/1 mм $\mathrm{MgCl}_{2} / 1 \mathrm{~mm} \mathrm{CaCl}_{2} / 35$ mм sucrose, $\mathrm{pH} 7.2$ ) supplemented with $10 \%$ Fetal Bovine Serum (Invitrogen), $50 \mathrm{U} / \mathrm{ml}$ penicillin (Invitrogen), $50 \mu \mathrm{g} / \mathrm{ml}$ streptomycin (Invitrogen), and $10 \mu \mathrm{g} / \mathrm{ml}$ amphotericin B (Invitrogen) as described previously (Wu et al., 1983). Briefly, brain and the ventral ganglion complex were dissected from Drosophila third instar larvae of the appropriate genotypes. The brain tissue was mechanically dissociated using syringe needles in Schneider's medium (Invitrogen) containing $0.75 \mu \mathrm{g} / \mu \mathrm{l}$ collagenase (Invitrogen) and $0.4 \mu \mathrm{g} / \mu \mathrm{l}$ dispase (Roche) and incubated in the proteolytic medium for 20 min to allow complete dissociation of the tissue. The lysate containing essentially single cells was spun down, resuspended in M1 medium (200 $\mu$ l of M1 was used for lysates of four brains), and plated onto polylysine-coated coverslips which formed the base of $35 \mathrm{~mm}$ culture dishes. The cells were incubated at $22^{\circ} \mathrm{C}$ for $14-16 \mathrm{~h}$ before imaging. All chemicals for cell culture were obtained from Sigma-Aldrich unless mentioned otherwise.

Calcium imaging in larval neurons. Calcium imaging in larval neuron cultures was performed after growth for 14-16 h. The cultures were washed with fresh Drosophila M1 medium twice and loaded in the dark with $2.5 \mu \mathrm{M}$ Fluo-4 acetoxymethyl ester (AM; Invitrogen) in M1 medium containing $0.002 \%$ Pluronic F-127 (Sigma-Aldrich), for $30 \mathrm{~min}$ at room temperature. After washing 3 times with M1, the cells were finally covered with $100 \mu \mathrm{l}$ of $\mathrm{Ca}^{2+}$-free $\mathrm{M} 1\left(\mathrm{CaCl}_{2}\right.$ was replaced with equal concentration of $\mathrm{MgCl}_{2}$ ) containing $0.5 \mathrm{~mm}$ EGTA and imaged within 40 min of loading. For quantitative analysis, a field with several cells was selected and imaged using the epifluorescence optics of a Nikon TE2000 inverted wide field microscope with an oil objective $(60 \times$ and $1.4 \mathrm{nu}-$ merical aperture) lens. Fluo- 4 was excited for $20 \mathrm{~ms}$ using $488 \mathrm{~nm}$ wavelength illuminations from a mercury arc lamp. Emitted light was detected through a $505 \mathrm{~nm}$ bandpass filter (FITC filter set, 41001-exciter HQ480/ 40, dichroic Q505LP, emitter HQ535/50; Chroma Technology Corp.). Fluorescent images were acquired using the Evolution QExi CCD camera and In Vivo imaging software (Media Cybernetics). The time lapse acquisition mode of the software was used to follow fluorescence changes in the cells every 10 or $15 \mathrm{~s}$ for 15 frames. Different concentrations of carbachol (Sigma-Aldrich), $10 \mu \mathrm{M}$ thapsigargin (Invitrogen), or $10 \mu \mathrm{M}$ ionomycin (Calbiochem, Merck) were added manually $\approx 15 \mathrm{~s}$ after the start of data acquisition. For measurement of store-operated $\mathrm{Ca}^{2+}$ entry, $1 \mathrm{mM} \mathrm{CaCl}_{2}$ was added to the cells $225 \mathrm{~s}$ after thapsigargin addition. Images were acquired every $15 \mathrm{~s}$. As controls, a series of images were acquired with the same imaging protocol without any additions. A total of 150-200 cells were imaged for each genotype for each experiment. All chemicals for calcium imaging were from Sigma-Aldrich unless mentioned otherwise.

Data analysis. For measuring fluorescence changes over time, images were processed using the ImagePro plus software, V1.33 (Media Cybernetics). Fluorescence intensity before $\left(F_{\text {basal }}^{\prime}\right)$ and at various time points after addition of carbachol, thapsigargin, or $\mathrm{CaCl}_{2}\left(F_{t}^{\prime}\right)$ were determined. Background fluorescence (an area without any cells) was subtracted from the values of $F_{t}^{\prime}$ and $F_{\text {basal }}^{\prime}$ for each cell to obtain $F_{t}$ and $F_{\text {basal }}$. The data were plotted using Origin 6.0 software as follows: $\Delta F / F=\left(F_{t}-F_{\text {basal }}\right) / F_{\text {basal }}$ for every time point. The maximum value of $\Delta F / F$ was obtained for every cell and a box chart representing the data spread was plotted. The rectangular 
A

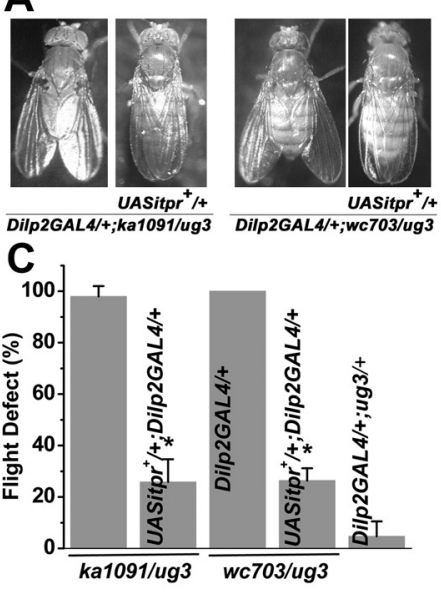

D

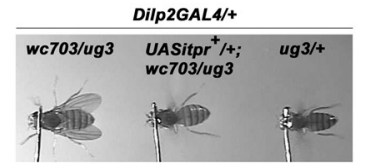

E

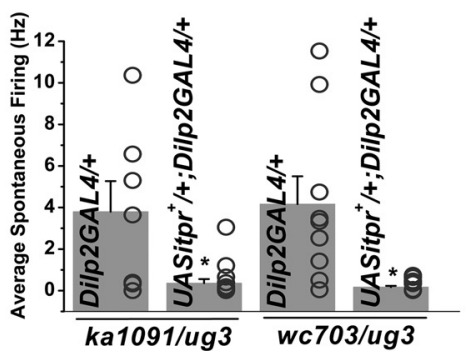

B *
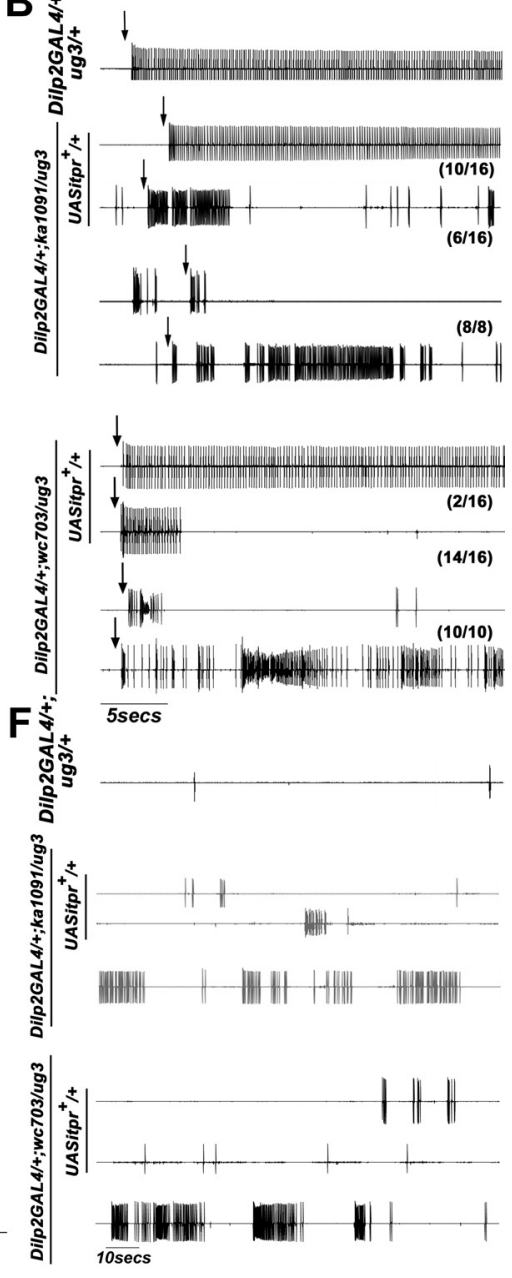

Figure 1. Flight and associated physiological defects in itpr mutant adults can be rescued by UASitpr ${ }^{+}$expression in DILP2 neurons. A, UASitpr ${ }^{+/+} ;$Dilp2GAL4/+ ;itpr ${ }^{\text {ka1091/ug3 }}$ and UASitpr ${ }^{+/+} ;$Dilp2GAL4/+;itpr $^{\text {wC703/ug3 }}$ flies exhibit normal wing posture compared with Dilp2GAL4/+;itpr ${ }^{\text {ka1091/ug3 }}$ and Dilp2GAL4/+;itpr ${ }^{\text {wC703/ug3 }}$ flies which have outspread wings. B, Electrophysiological recordings from the DLMs of tethered flies after delivery of an air puff stimulus (arrows). Dilp2GAL4 rescued flies can respond with a rhythmic pattern that is sustained for the entire 30 s duration of the recording similar to control flies of the genotype Dilp2GAL4/+;itpr ${ }^{u g 3} /+$. A higher proportion of UASitpr ${ }^{+/+} ;$Dilp2GAL4/+,itpr ${ }^{\text {ka1091/ug3 }}$ adults showed sustained flight pattern (10/16) in comparison with UASitpr ${ }^{+/+} ;$Dilp2GAL4/+;itpr $^{\text {wC703/ug3 }}(2 / 16)$. Air puff-induced flight patterns are absent in itpr

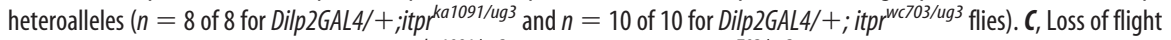
assayed by the cylinder drop test seen in itpr ${ }^{k a 1091 / u g 3}$ and Dilp2GAL4/+,itpr ${ }^{w c 703 / u g 3}$ is significantly rescued by expression of UASitpr $^{+}$with Dilp2GAL4 (* $p<0.005$; Student's $t$ test). Approximately 100 or more flies were tested for each genotype. Results are expressed as mean \pm SEM. $D$, Single frames from movies of air puff-induced flight in single tethered flies of the indicated genotypes (see also supplemental Video 1, available at www.jneurosci.org as supplemental material). Wings are not visible in the rescued and control condition as they are beating. $\boldsymbol{E}$, Quantification of spontaneously generated action potentials. The number of spikes observed in recordings over 2 min were counted and averaged from flies of the indicated genotypes to obtain individual firing frequencies in $\mathrm{Hz}$ (individual data points are represented by open circles). The spontaneous firing rate in $U_{A S i t p r}{ }^{+/+}$; Dilp2GAL4/+, ;itpr ${ }^{\text {Ka 1091/ug3 }(n=17) \text { and UASitpr }}{ }^{+/+} ; \operatorname{Dilp2GAL4/+;itpr}^{w c 703 / u g 3}(n=16)$ flies is significantly reduced compared with Dilp2GAL4/+,itpr ${ }^{\text {ka1091/ug3 }}(n=7)$ and Dilp2GAL4/+,itpr ${ }^{\text {wc703/ug3 }}(n=9)\left(^{*} p<0.005\right.$; Student's $t$ test). Results are expressed as mean \pm SEM. $\boldsymbol{F}$, Representative traces of spontaneously generated action potentials observed in recordings from the DLMs of indicated itpr mutants and in Dilp2GAL4 rescued flies.

boxes represent the spread of data points between $25 \%$ and $75 \%$ of cells, the horizontal line is the median, and the small square within represents the mean. Significant differences between multiple groups of data were analyzed by one-way ANOVA test of significance as indicated in the figure legends. Significant differences were taken at $P_{\text {ANOVA }}<0.05$.

\section{Results}

Restoring $i t \mathrm{pr}^{+}$function in DILP2 neurons rescues flight defects of itpr mutants

Expression of the wild-type Drosophila InsP ${ }_{3} \mathrm{R}$ cDNA (UASitpr ${ }^{+}$) in the aminergic domain can rescue both larval and adult pheno-

types of itpr mutants (Banerjee et al., 2004; Joshi et al., 2004). Larval itpr mutant phenotypes of growth, feeding and viability can also be rescued to a significant extent by $\mathrm{itpr}^{+}$expression in the DILP2 neurons (Agrawal et al., 2009). To investigate the effect of restoring itpr function in the DILP2 neurons on adult itpr mutant phenotypes, UASitpr ${ }^{+}$was expressed with Dilp2GAL4 (Rulifson et al., 2002) in adult viable itpr mutant heteroallelic combinations, itpr ${ }^{k a 1091 / u g 3}$ and $i t p r^{\text {wc703/ug } 3}$. $i t p r^{k a 1091}$ and $i t p r^{w c 703}$ alleles each have single point mutations in the modulatory domain of the $\operatorname{InsP}_{3} \mathrm{R}$, while $i t p r^{u g 3}$ has a single point mutation in the $\mathrm{N}$-terminal ligand binding domain of the $\operatorname{Ins}_{3} \mathrm{R}$ (Joshi et al., 2004). Surprisingly, complete rescue of the altered wing posture in $i_{\text {tpr }}$ ka1091/ug3 $^{2}$ and $i t p r^{\text {wc703/ug } 3}$ was seen in UASitpr $^{+/+} ;$Dilp2GAL4/+;itpr ${ }^{\text {kalogl/ugs } 3}$ and UASitpr $^{+/+} ;$Dilp2GAL4/+;itpr ${ }^{\text {wc703/ug3 }}$ animals (Fig. 1A). Moreover, flight ability was rescued significantly as measured by the "cylinder drop test" assay (Fig. 1C). Flight defects are not significantly different between males and females of either itpr mutants or of itpr mutants expressing UASitpr $^{+}$with the Dilp2GAL4 (data not shown). The physiological correlate of loss of flight in itpr $r^{\text {ka1091/ug3 }}$ and $i t p r^{\text {wc703/ug } 3}$ flies is an inability to initiate a rhythmic train of action potentials in response to an air-puff stimulus and a considerably higher frequency of spontaneous action potentials as measured by electrophysiological recordings from the dorsal longitudinal set of indirect flight muscles (DLMs) (Banerjee et al., 2004). The delivery of an air puff stimulus to $i_{t p p} r^{k a 1091 / u g 3}$ flies expressing UASitpr ${ }^{+}$with the Dilp2GAL4 led to a rhythmic generation of action potentials similar to what is observed in wild-type flies (Fig. $1 B$ ). Though, a majority of the flies tested had a sustained air puff response (10/16), in some flies $(6 / 16)$ the response was more erratic and could not be maintained for more than five seconds. $i t p r^{w c 703 / u g 3}$ flies expressing UASitpr $^{+}$with the Dilp2GAL4 also led to rhythmic generation of action potentials immediately after the delivery of an air puff stimulus, but in most flies (14/16) the air puff response lasted for a shorter period of time compared with the control flies (Fig. $1 B, D$; supplemental Video 1, available at www.jneurosci.org as supplemental material). Increased spontaneous firing from the DLMs, which is characteristic of $i t p r^{k a 1091 / u g 3}$ and $i t p r^{\text {wc703/ug } 3}$, was also significantly reduced in the Dilp2GAL4 rescued condition (Fig. 1E,F). Flight and associated physiology was not rescued in itpr mutants by expression of UASitpr ${ }^{+}$in neuropeptidergic domains other than Dilp2GAL4. The neuropeptidergic domains tested were P386GAL4 (Taghert et al., 2001), c929GAL4 (Hewes et al., 2003), AKHGAL4 (Kim and Rulifson, 2004) and CCAPGAL4 (Park et al., 2003; data not shown). 
These data suggest that DILP2 neurons can participate in the development and/or function of the air-puff stimulated flight circuit. Wing posture, flight and flight physiology can be rescued by ubiquitous expression of the wild-type itpr transgene during pupal development (Banerjee et al., 2004). To address the question of itpr gene requirement in the DILP2 neurons during pupal development the TARGET (temporal and regional gene expression targeting) system (McGuire et al., 2003) was used for regulating the temporal expression of UASitpr ${ }^{+}$in the Dilp2GAL4 domain of itpr $r^{k a 1091 / u g 3}$ organisms. The TARGET system includes a temperaturesensitive GAL80 element $\left(G A L 80^{t s}\right)$ that regulates GAL4 in a temperature-dependent manner, with optimal repression observed at $19^{\circ} \mathrm{C}$ and derepression at $30^{\circ} \mathrm{C}$ (McGuire et al., 2004). Wing posture and air puff-induced flight (assessed in single fly tethered assays) were not rescued when UASitpr ${ }^{+/+}$; Dilp2GAL4/GAL80 ${ }^{\text {ts }}$; itpr $^{\text {ka1091/ug3 }}$ animals were grown at $19^{\circ} \mathrm{C}$ during pupal and adult stages (Fig. 2A), but were completely rescued when these animals were grown at $30^{\circ} \mathrm{C}$ (Fig. $2 \mathrm{~B}$; supplemental Video 2, available at www. jneurosci.org as supplemental material). UASitpr $^{+/+}$;Dilp2GAL4/GAL80 ${ }^{\text {ts }}$ itpr $r^{\text {ka1091/ug3 }}$ organisms, grown at $19^{\circ} \mathrm{C}$ through pupation and shifted to $30^{\circ} \mathrm{C}$ as adults, also had defective wing posture and flight ability (Fig. 2C; supplemental Video 3, available at www. jneurosci.org as supplemental material). However, UASitpr ${ }^{+/+}$;Dilp2GAL4/GAL80 $0^{t s}$; itpr $^{\mathrm{ka1091/ug3}}$ organisms, grown at $30^{\circ} \mathrm{C}$ during pupal stages but shifted to $19^{\circ} \mathrm{C}$ as adults, had normal wing posture and air puff-induced flight (Fig. 2D; supplemental Video 4, available at www.jneurosci.

org as supplemental material) indicating a requirement for $i t p r$ expression in the DILP2 neurons during pupal development, when the flight circuit is formed. An additional requirement during acute adult flight remains possible due to perdurance of the Ins $\mathrm{P}_{3} \mathrm{R}$ in DILP2 neurons from pupae to adults.

\section{Overexpression of the ER store calcium sensor $d S$ TIM $^{+}$in DILP2 or aminergic neurons suppresses flight defects of $i t p r$ mutants}

Certain adult flight phenotypes in itpr $r^{k a 1091 / u g 3}$, such as wing posture, spontaneous firing frequency and flight initiation can be partially suppressed by expression of the wild-type cDNA for the SOC $\mathrm{dOrai}^{+}$in DILP2 and aminergic neurons (Venkiteswaran and Hasan, 2009). Together with the rescue by itpr ${ }^{+}$expression in these domains and its requirement during pupal development, the data suggest that $\mathrm{Ca}^{2+}$ release through the Ins $\mathrm{P}_{3} \mathrm{R}$ and storeoperated $\mathrm{Ca}^{2+}$ entry (SOCE) in DILP2 and aminergic neurons influence Drosophila flight circuit development to a significant extent.

The partial suppression of flight phenotypes by $\mathrm{dOrai}^{+}$may be due to insufficient levels of the ER $\mathrm{Ca}^{2+}$ sensor STIM which
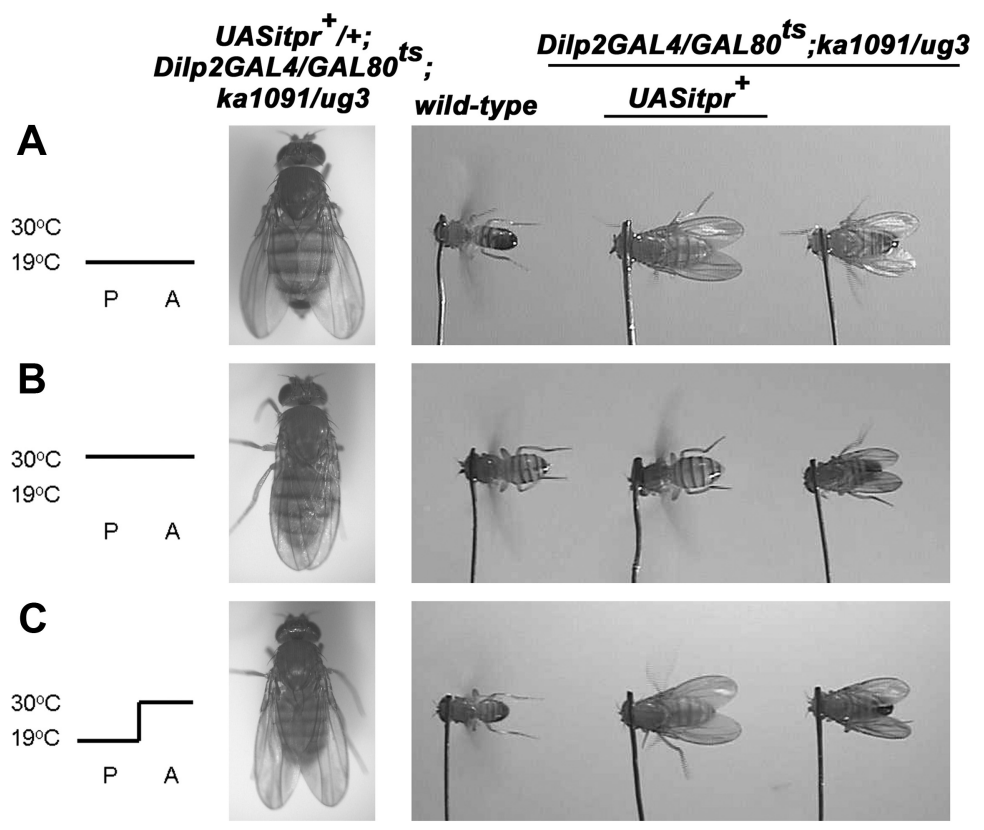

Figure 2. Adult itpr mutant phenotypes can be rescued by expression of UASitpr ${ }^{+}$in DILP2 neurons during pupal

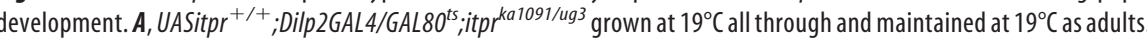
The adults exhibit defective wing posture and cannot initiate wing beating in response to an air-puff stimulus $(0 / 2$ individual flies tested) similar to Dilp2GAL4/GAL80 ${ }^{t 5}$; itpr ${ }^{k a 1091 / u g 3}$ flies on the right and unlike wild-type flies on the left. 列

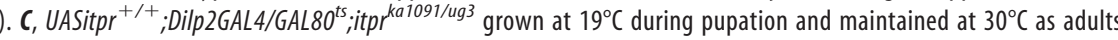
a defective wing posture and cannot initiate wing beating in response to an air-puff stimulus (0/12 individual flies Dilp2GAL4/GAL80 ts. itpra1091/ug 3 grown at $30^{\circ} \mathrm{C}$ during pupation and maintained at $19^{\circ} \mathrm{C}$ as adults have a normal wing posture and can initiate wing beating in response to an air-puff stimulus (9/10 individual flies tested; see also supplemental Video 4 , available at www.jneurosci.org as supplemental material).

works together with Orai to bring about SOCE (Cahalan, 2009). Coexpression of Orai and STIM results in SOCE which is significantly greater than that observed by the expression of either protein alone (Soboloff et al., 2006). This idea was tested further by coexpressing $d S T I M^{+}$and $d \mathrm{Orai}^{+}$in the DILP2 and aminergic neurons of $i t p r^{k a 1091 / u g 3}$ organisms. For this purpose transgenic strains were generated with $d S T I M^{+}$. Coexpression of $d S T I M^{+}$ and $\mathrm{dOrai}^{+}$in DILP2 neurons completely suppressed the wing posture, flight and flight physiology defects of $i t p r^{k a 1091 / u g 3}$ (Fig. $3 A-E$; supplemental Video 5, available at www.jneurosci.org as supplemental material). Simultaneous expression of $d S T I M^{+}$ and $\mathrm{dOrai}^{+}$in aminergic neurons resulted in pupal lethality (data not shown). Interestingly, expression of $d S T I M^{+}$alone in DILP2 or aminergic neurons also suppressed the altered wing posture of $i_{\text {tpr }}^{\text {ka1091/ug3 }}$ flies (Fig. 3A) as well as spontaneous hyperactivity of the DLMs (Fig. $3 D, E$ ). Importantly, expression of $d S T I M^{+}$in either DILP2 or aminergic neurons of $i t p r^{k a 1091 / u g 3}$ flies restored flight ability and sustained air-puff-induced rhythmic flight patterns (Fig. 3B,C). This rescue of itpr mutant phenotypes was better than that observed on expression of $\mathrm{UASdOrai}^{+}$in these domains, where a partial rescue of wing posture and a brief initi- 
A

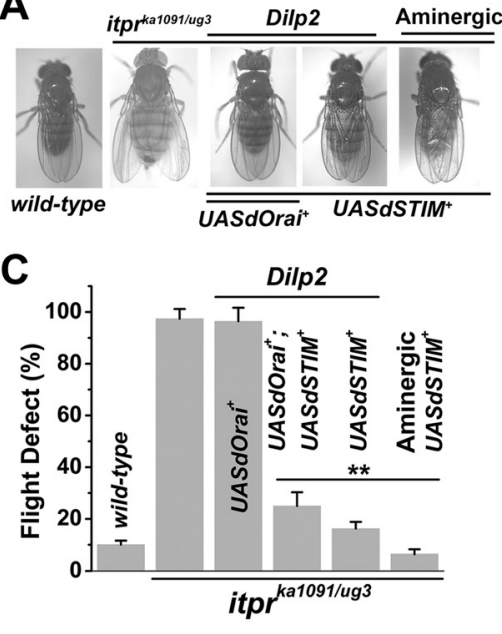

D

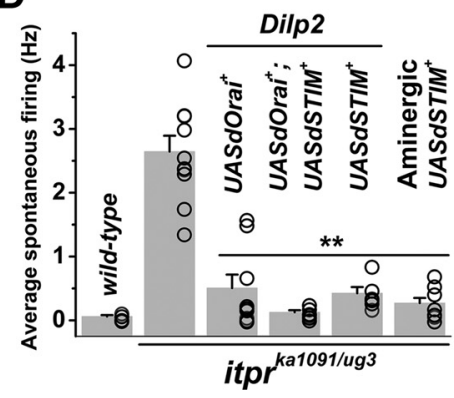

B
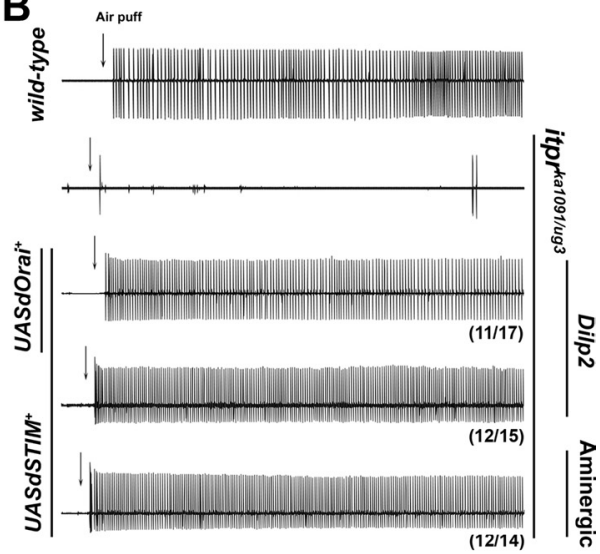

5 secs

E

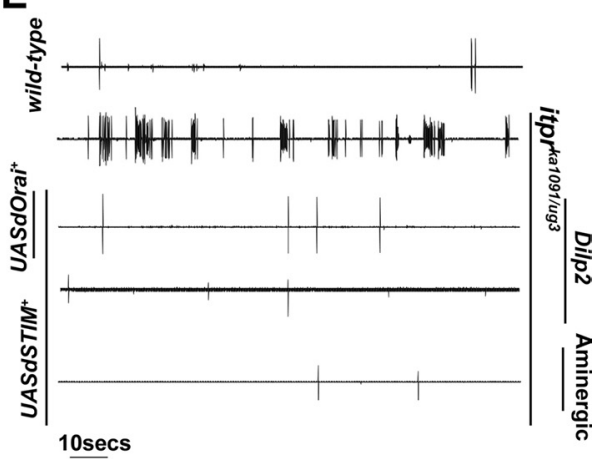

Figure 3. Expression of $d S T I M^{+}$in the DILP2 neurons suppresses flight defects of itpr ${ }^{k a 1091 / u g 3}$. A, Expression of dSTIM ${ }^{+}$ and $\mathrm{dOrai}^{+}$simultaneously with Dilp2GAL4 (Dilp2) or dSTIM ${ }^{+}$alone with Dilp2GAL4 or DdCGAL4 (Aminergic) suppresses the wing posture defect of $i t p r^{\text {ka1091/ug3 }}$. B, Representative traces of flight patterns from DLMs after delivery of an air-puff stimulus (arrow).Targeted expression of either dOrai ${ }^{+}$and SSTIM $^{+}$or dSTIM ${ }^{+}$on its own, with Dilp2GAL4 or DdCGAL4 in $i_{\text {itpr }}^{k a 1091 / u g 3}$ flies, elicits sustained rhythmic action potentials on delivery of an air-puff, in DLM recordings. C, Complete suppression of the flight defect in itpr ${ }^{\text {ka1091/ug } 3}$ is observed upon expression of dSTIM ${ }^{+}$with Dilp2GAL4 or DdCGAL4 $\left(^{* *} p<\right.$ 0.01; Student's $t$ test), not seen upon expression of $d_{0} \mathrm{rai}^{+}$alone (Venkiteswaran and Hasan, 2009). D, Frequency of spontaneous firing recorded from the DLMs of itpr ${ }^{\mathrm{ka}^{1091 / u g 3}}$ is significantly reduced upon expression of UASdOrai ${ }^{+}$and/or UASdSTIM $^{+}$with Dilp2GAL4 or DdcGAL4 in itpr ${ }^{\text {ka1091/ug } 3}$ flies ( ${ }^{* *} p<0.01$; Student's $t$ test). $\boldsymbol{E}$, Representative traces showing suppression of spontaneous hyperactivity by targeted expression of UASdOrai ${ }^{+}$and/or UASdSTIM ${ }^{+}$using Dilp2GAL4 or DdCGAL4.

ation of air-puff-induced flight was obtained (Venkiteswaran and Hasan, 2009). These results suggest that $d S T I M^{+}$expression on its own can efficiently restore intracellular calcium homeostasis in DILP2 neurons, and this restoration can bring about systemic suppression of itpr mutant phenotypes.

Pan-neuronal expression of $d \mathrm{Orai}^{+}$and $d S T I M^{+}$in itpr ${ }^{\text {kalog1/ug3 }}$ organisms suppresses wing-posture to varying extents but does not restore flight ability to levels comparable with Dilp2GAL4 and DdcGAL4 driven expression (supplemental Fig. 1, available at www.jneurosci.org as supplemental material). These data are consistent with previous observations that flight in itpr $r^{k a 1091 / u g} 3$ is not rescued by pan-neuronal expression of $i t p r^{+}$with the ElavGAL4 (Banerjee et al., 2004). Expression of $i t p r^{+}$with Elav ${ }^{\text {C155 GAL4 }}$ (which has a stronger expression in the pupal stages than ElavGAL4; data not shown) can partially rescue wing posture and flight physiology defects but is insufficient for restoring flight ability (supplemental Fig. 1, available at www.jneurosci.org as supplemental material). Differences in temporal and spatial levels of pan-neuronal GAL4 expression coupled with a differential requirement for $\mathrm{Ins}_{3} \mathrm{R}$ function at multiple steps of flight circuit formation are a likely explanation for these data (see Discussion and Banerjee et al., 2006).

\section{Relation between the Dilp2GAL4 and}

\section{Ddc domains}

The simplest explanation for the rescue of itpr mutant phenotypes by expression of $U$ ASitpr $^{+}$or UASdSTIM $^{+}$in aminergic and DILP2 neurons is an overlap between the two domains. However in larvae, where the two domains have a similar effect upon rescue of growth and viability, DILP2 and aminergic neurons do not overlap (Agrawal et al., 2009). To determine whether an overlap develops at later stages, a membrane bound GFP (UASmCD8GFP) was expressed with Dilp2GAL4 and pupal and adult brains of these animals were stained with an anti-Ddc antibody (Lundell and Hirsh, 1994).

Since the rescue by Dilp2GAL4 has a temporal focus during pupal development, a possible overlap of DILP- and Ddc-positive cells in the CNS of $36 \mathrm{~h} \mathrm{pu-}$ pae was investigated (Fig. 4). No overlap between Ddc cells and the DILP2 neurons was observed in either the developing pupal brain (Fig. $4 A-C$ ) or the ventral ganglion (Fig. $4 D-F$ ). A cluster of Ddcpositive cells (Fig. 4A, red arrowheads) were present in close proximity to the DILP2 neurons and their processes project in close proximity to processes from insulin-producing cells. A cluster of Ddc-positive cells was observed in the subesophageal ganglion (Fig. 4A, red arrows) which are also seen in larval (Agrawal et al., 2009) and adult brains. However, no cells positive for both GFP and Ddc could be seen in the thoracic and abdominal ganglia, though a cluster of Dilp2::GFP cells in the thoracic ganglia (Fig. $4 E$, green arrowhead) had processes extending toward (Fig. $4 E$, green arrows) segmentally labeled Ddc cells in the abdominal ganglia (Fig. $4 D$, red arrowhead). The overlap of DdcGAL4 expression with the Ddc antibody used here was also ascertained. A few cells in the $D d c G A L 4$ strain did not stain with anti-Ddc (supplemental Fig. 2, available at www.jneurosci.org as supplemental material). However, none of these were in the vicinity of the Dilp2GAL4-positive cells.

In the adult brain too, no overlap between Ddc and DILP2 neurons (Fig. 5C, green arrowhead) was observed (Fig. 5D, $H$, white arrowheads). Similar to observations in the developing pupal brain, Ddc-positive cells on the lateral part of the subesophageal ganglion (Fig. 5F, J, red arrowheads) had processes that extended toward the medial part of the subesophageal region (Fig. $5 \mathrm{~F}$, J, red arrows) in close proximity to where processes from DILP2 neurons terminate (Fig. 5C,G,K, green arrows). Though $D d c G A L 4$ expresses in both serotonergic and dopaminergic neurons, itpr mutant phenotypes are not rescued by expression of UASitpr $^{+}$in the dopaminergic domain (with the THGAL4; Friggi-Grelin et al., 2003; our unpublished data) suggesting that rescue of itpr mutants is through serotonergic neurons in the context of the phenotypes under study. These lateral Ddcpositive cells also stained with the anti-serotonin antibody (Fig. 
5I, blue arrowheads). Serotonin-positive varicosities were visible near the DILP2 neurons and on the processes extending from them (Fig. 5E, blue arrows). The thoracic and the abdominal ganglia did not show any cells labeled with both GFP and Ddc. These experiments indicate the absence of any detectable overlap between Dilp2 and DdcGAL4 domains in the CNS of pupae and adults.

\section{Pan-neuronal but not tissue-specific knockdown of itpr, dSTIM, or dOrai recapitulates adult itpr mutant phenotypes}

The rescue of flight and associated defects observed in itpr mutants independently by either Dilp2GAL4 and DdcGAL4 and the absence of any detectable overlap between them suggests the existence of nonoverlapping neuronal domains that might operate in parallel to regulate flight circuit development. To further understand the requirement of $\mathrm{Ca}^{2+}$ homeostasis in these domains for flight ability, specific knockdown of itpr, dSTIM and dOrai was attempted.

Ubiquitous reduction of $\mathrm{Ins}_{3} \mathrm{R}$ levels by expression of a dsitpr construct with the Actin5cGAL4 line is larval lethal, while itpr knockdown in either Elav ${ }^{C 155}$ GAL4, Dilp2GAL4 or DdcGAL4 domains had no observable effect on larval viability (Agrawal et al., 2009). Panneuronal knockdown of itpr however results in partial pupal le-

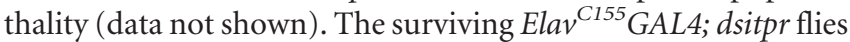
exhibit wing posture defects (Fig. 6A), reminiscent of those observed in adult viable itpr heteroallelic mutant combinations. The spread out wing posture defect was further exacerbated on enhancing RNA interference by expressing a UASdicer transgene (Fig. 6A). Moreover, the wing posture defect was more pronounced in males compared with females, very likely due to differences in dosage compensation of Elav ${ }^{C 155}$ GAL4, an insert on the first chromosome (Fig. 6A). Elav ${ }^{C 155}$ GAL4; dsitpr flies exhibit significant levels of flight defects (Fig. $6 B$ ) and were unable to initiate rhythmic flight patterns in recordings from the DLMs in response to an air-puff stimulus (0/12; Fig. 6C).

However, no significant change in wing posture or flight ability was observed when dsitpr was expressed with either Dilp2GAL4 or $D d c G A L 4$ (Fig. 6A,B) even when dsitpr expression was enhanced with a dicer transgene. A majority of these flies show a sustained air-puff response (Fig. 6C). Nevertheless, increased spontaneous firing from the DLMs was observed on both pan-neuronal or tissue-specific knockdown of itpr, suggesting that neuronal activity in the flight circuit is mildly perturbed due to reduced $\operatorname{Ins}_{3} \mathrm{R}$ function in either DILP2 or aminergic neurons (Fig. $6 D, E$ ). The effect of itpr knockdown simultaneously in both DILP2 and Ddc domains was tested by generating a recombinant strain from the Dilp2GAL4 and DdcGAL4 strains. This strain is referred to as DoubleGAL4 in Figure 6. Adult flies eclosed normally in the genotype with doubleGAL4 driven expression of dsitpr enhanced by UASdicer. The adults had normal wing posture (data not shown) and passed the flight column test (Fig. 6B). Electrophysiological recordings from the DLMs of these individuals exhibit a high rate of spontaneous firing as in the case of the individual GAL4s (Fig.
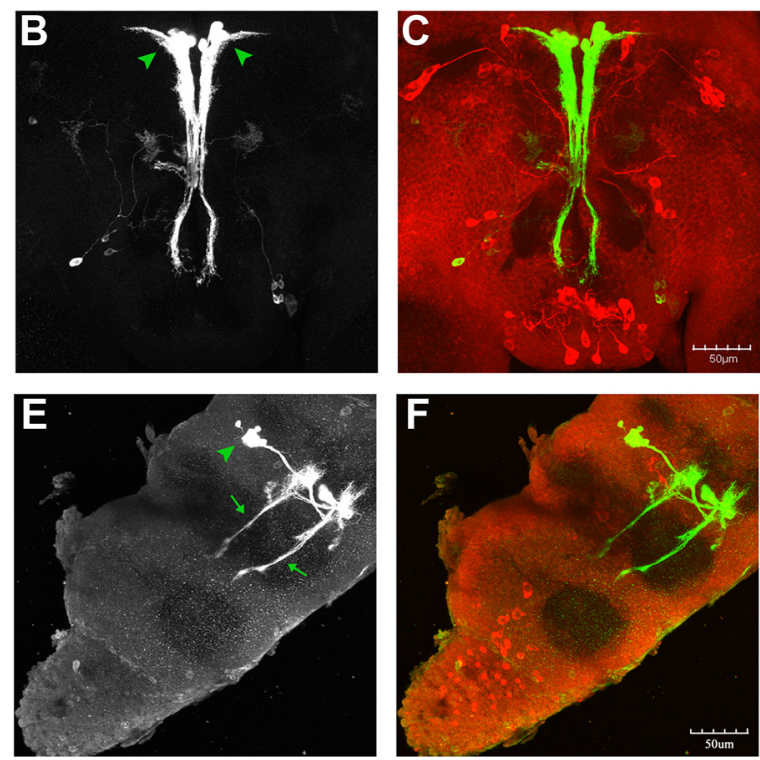

Figure 4. Ddc-labeled cells and DILP2 neurons do not overlap in pupal brains. $\boldsymbol{A}-\boldsymbol{F}$, Projections of confocal Z-stacks of a arrowheads in $\boldsymbol{E}$ mark one of a pair of anti-GFP-labeled clusters in the ganglia that sends out processes toward segmentally labeled Ddc cells in the abdominal ganglia (red arrowheads in $\boldsymbol{D}$ ). Scale bars $(\boldsymbol{A}-\boldsymbol{F}), 50 \mu \mathrm{m}$.

$6 D, E)$. However, unlike the individual $G A L 4 s, 50 \%$ of flies in which the dsitpr transgene was driven by the double GAL4 strain, were unable to maintain flight beyond 15-20 s (Fig. 6C). Presumably, this level of flight maintenance is sufficient for passing the flight column test. Thus the combined knockdown of itpr in DILP2 and Ddc domains affects flight circuit function to a greater extent than knock down in the individual domains.

Pan-neuronal knockdown of either $d S T I M$ or dOrai results in defects in wing posture, flight and flight physiology (Venkiteswaran and Hasan, 2009). However, in observations similar to those obtained on expression of dsitpr, no wing posture defects were observed on expression of $d s d S T I M$ or $d s d O r a i$ with either the Dilp2GAL4 or DdcGAL4 (supplemental Fig. 3A, available at www. jneurosci.org as supplemental material). Moreover, the flight ability of these flies was not significantly different from controls (supplemental Fig. 3B, available at www.jneurosci.org as supplemental material). These results support the existence of multiple neural domains that can contribute in parallel to flight circuit development. Perturbing $\mathrm{Ca}^{2+}$ homeostasis in one or two neuronal domains may thus be insufficient for inducing strong flight defects.

Next, the nature of alterations in intracellular calcium signaling was investigated among the various genotypes studied. Panneuronal downregulation of the $\operatorname{InsP}_{3} \mathrm{R}$ by dsRNA is expected to reduce $\mathrm{InsP}_{3}$-mediated $\mathrm{Ca}^{2+}$-release as demonstrated for itpr mutant neurons. In addition, certain itpr mutants also show global dysregulation of intracellular $\mathrm{Ca}^{2+}$ homeostasis by altering $\left[\mathrm{Ca}^{2+}\right]_{\mathrm{ER}}$ and SOCE (Venkiteswaran and Hasan, 2009). To assess $\mathrm{Ca}^{2+}$ homeostasis on pan-neuronal downregulation of itpr, $\left[\mathrm{Ca}^{2+}\right]_{\mathrm{ER}}$ and SOCE was measured in primary neuronal cultures obtained from brains of Elav ${ }^{C 155}$ GAL4; dsitpr larvae (Fig. $6 \mathrm{~F}-\mathrm{H})$. SOCE was monitored by $\mathrm{Ca}^{2+}$ imaging in $\mathrm{Ca}^{2+}$ addback experiments, after depletion of ER stores with thapsigargin in very low external $\mathrm{Ca}^{2+}$. SOCE was reduced in neurons expressing dsRNA for itpr compared with controls (Fig. 6F-H, 

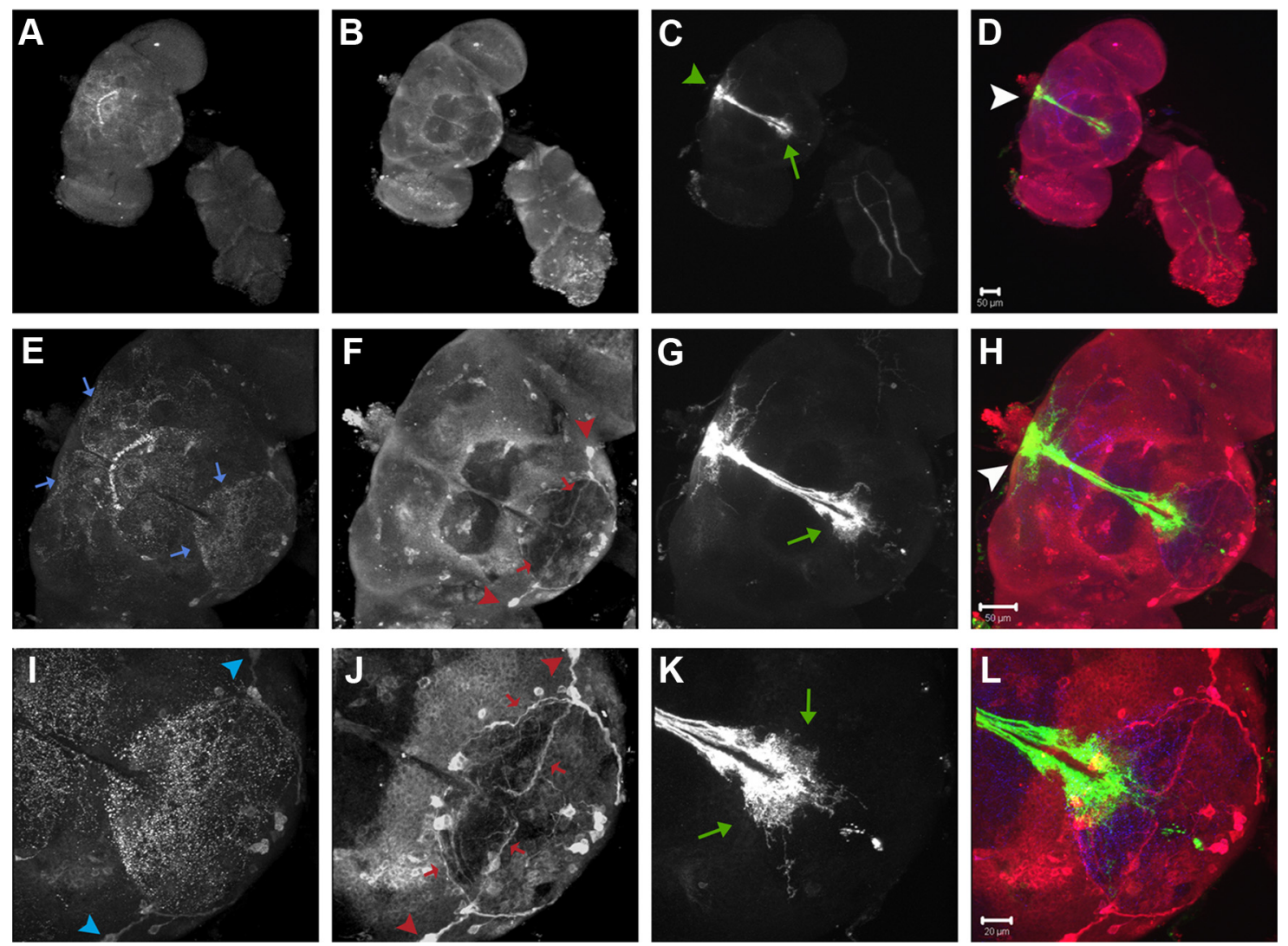

Figure 5. Ddc-and serotonin-labeled cells in larval and adult brains do not overlap with the DILP2 neurons. $A-L$, Projections of confocal Z-stacks of a wild-type Drosophila adult brain expressing mCD8GFP with Dilp2GAL4 and immunostained with anti-serotonin antibody $(\boldsymbol{A}, \boldsymbol{E}, \boldsymbol{I})$, anti-Ddc antibody $(\boldsymbol{B}, \boldsymbol{F}, \boldsymbol{J})$ and anti-GFP antibody $(\boldsymbol{C}, \boldsymbol{G}, \boldsymbol{K})$. $\boldsymbol{D}, \boldsymbol{H}$, and $\boldsymbol{L}$ are a merge of the corresponding images with anti-serotonin labeling in blue, anti-Ddc labeling in red, and anti-GFP labeling in green. Successive rows are higher-magnification images. Top and bottom blue arrows in $\boldsymbol{E}$ indicate serotonin-positive varicosities near the DILP2 neurons and in the subesophageal ganglia, respectively. Blue arrowheads in $\boldsymbol{I}$ and red arrowheads in $\boldsymbol{F}$ and $\boldsymbol{J}$ mark cells that are Ddc and serotonin positive and send out processes centrally toward the subesophageal ganglia (red arrows in $\boldsymbol{F}, \boldsymbol{J}$ ) that seem to intermingle with the processes sent out by the DILP2 neurons (green arrows in $\boldsymbol{C}, \boldsymbol{G}, \boldsymbol{K}$ ). Green arrowhead in $\mathbf{C}$ and white arrowheads in $\boldsymbol{D}, \boldsymbol{H}$ mark the DILP2 neurons. Scale bars: $\boldsymbol{A}-\boldsymbol{H}, 50 \mu \mathrm{m} ; \boldsymbol{I}-\mathbf{L}, 20 \mu \mathrm{m}$.

$\left.P_{\text {ANOVA }}<0.05\right)$ similar to the results obtained with panneuronal expression of $d s d S T I M$ or $d s d O r a i$ (Venkiteswaran and Hasan, 2009). However, store $\mathrm{Ca}^{2+}$ or $\left[\mathrm{Ca}^{2+}\right]_{\mathrm{ER}}$ was similar between Elav ${ }^{C 155}$ GAL4; dsitpr neurons and controls (Fig. $6 \mathrm{H}$ ). These data suggest that the primary effect of dsitpr is likely to be on the magnitude of $\mathrm{Ins}_{3}$-mediated $\mathrm{Ca}^{2+}$-release, followed by a milder change in SOCE.

\section{Global restoration of calcium homeostasis on tissue-specific} expression of itpr $^{+}$in itpr $^{\mathrm{ka1091/ug3}}$

So far, restoration of $i t p r^{+}$activity in a small neuronal subset in an itpr mutant background is sufficient for systemic rescue of the observed physiological phenotypes. In neurons derived from itpr $r^{\text {ka1091/ug3 }}$ multiple parameters of intracellular $\mathrm{Ca}^{2+}$ homeostasis including the magnitude of $\mathrm{Ca}^{2+}$ released through InsP ${ }_{3} \mathrm{R}, \mathrm{SOCE}$ and store $\mathrm{Ca}^{2+}$ are perturbed (Venkiteswaran and Hasan, 2009). In the rescued organisms, restoration of $\mathrm{Ca}^{2+}$ homeostasis in either the DILP2 or aminergic neurons might be sufficient for the observed systemic rescue. Alternatively, itpr $r^{+}$ expression in these subneuronal domains may restore global $\mathrm{Ca}^{2+}$ homeostasis by non-cell-autonomous mechanisms. To delineate between these two possibilities, store $\mathrm{Ca}^{2+}$ and SOCE were measured in cultured neurons derived from larval brain and ventral ganglion complex in conditions where $i t p r^{+}$expression was either pan-neuronal or in DILP2 or aminergic neurons.

Pan-neuronal expression of $\mathrm{itpr}^{+}$in an itpr $\mathrm{ka1091/ug3}^{3}$ background restored both SOCE and store $\mathrm{Ca}^{2+}$ to levels seen in wild-type (Fig. 7C,D). Interestingly, SOCE and store $\mathrm{Ca}^{2+}$ in neurons from $i t p r^{k^{k 1091 / u g 3}}$ larvae expressing $i t p r^{+}$in either DILP2 or aminergic neurons was also similar to wild-type (Fig. 7A-D). The proportion of cells exhibiting detectable SOCE was restored upon expression of UASitpr ${ }^{+}$under Ddc (68\%) or Dilp2GAL4 (71\%) compared with itpr ka1091/ug3 (<5\%; Table 1). These results strongly suggest that $\mathrm{Ca}^{2+}$ signaling through the $\mathrm{InsP}_{3} \mathrm{R}$ in DILP2 and aminergic neurons helps maintain global intracellular $\mathrm{Ca}^{2+}$ homeostasis through non-cell-autonomous mechanisms.

\section{Overexpressing $d S T I M^{+}$in itpr $r^{k a 1091 / u g 3}$ DILP2 neurons} restores global intracellular $\mathrm{Ca}^{2+}$ homeostasis

To understand the cellular basis of suppression of $i t p r^{k a 1091 / u g 3}$ phenotypes by SSTIM $^{+}$expression in the DILP2 neurons, SOCE and store $\mathrm{Ca}^{2+}$ were measured in larval neuronal cultures generated from larvae expressing $\mathrm{dSTIM}^{+}$or $\mathrm{dOrai}^{+}$either individually or by coexpressing both transgenes in DILP2 neurons. SOCE was detectable in $\sim 75 \%$ of neurons upon overexpression either $\mathrm{dOrai}^{+}$or $\mathrm{dSTIM}^{+}$or both using Dilp2GAL4. The extent of SOCE and store $\mathrm{Ca}^{2+}$ in these neuronal populations was signif-

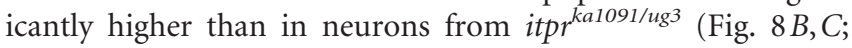
$\left.P_{\text {ANOVA }} \ll 0.05\right)$. In comparison with wild-type, SOCE remained significantly lower in neurons from itpr $^{k a 1091 / u g 3}$ larvae expressing $d \mathrm{Orai}^{+}$in DILP2 neurons $\left(P_{\text {ANOVA }}<0.05\right)$. In contrast, the extent of SOCE and store $\mathrm{Ca}^{2+}$ was similar to wild-type $\left(P_{\text {ANOVA }}>\right.$ $0.05)$, in the neuronal population on expression of $d S T I M^{+}$in DILP2 neurons. Thus, expression of SSTIM $^{+}$in DILP2 neurons 


\section{A}

Control

Pan-neuronal

Aminergic Dilp2
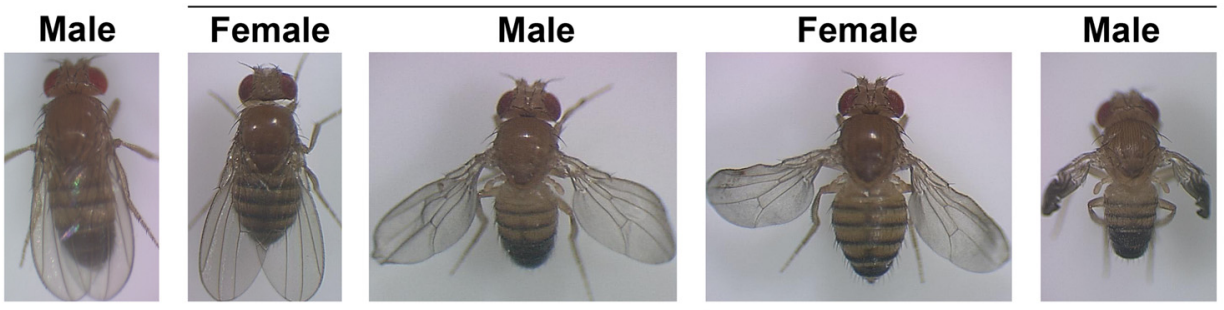

Female Female

\section{dsitpr}

dicer

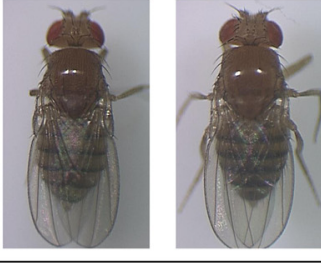

C

B

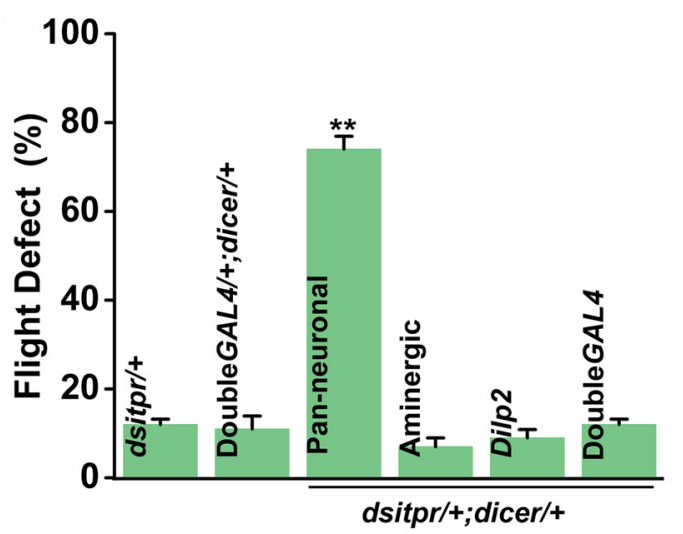

D

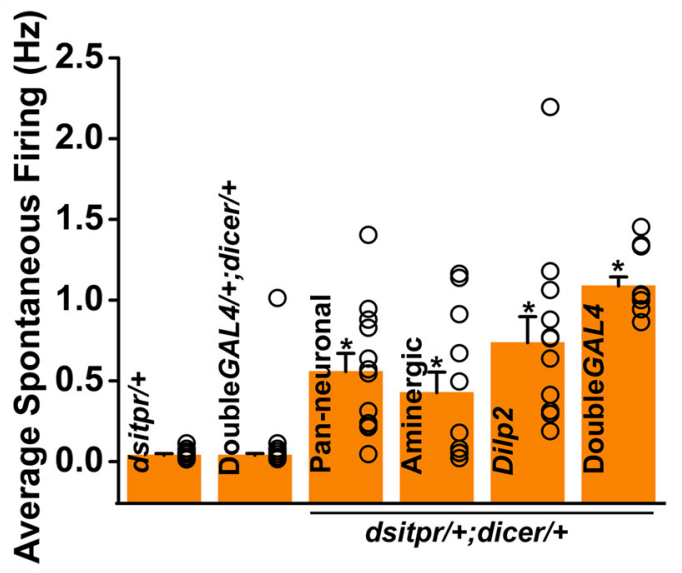

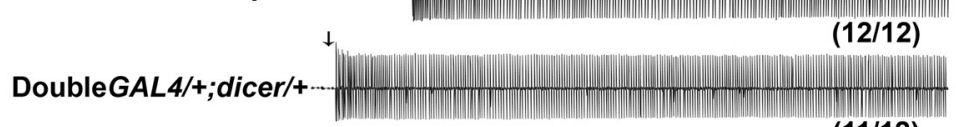

(11/12)

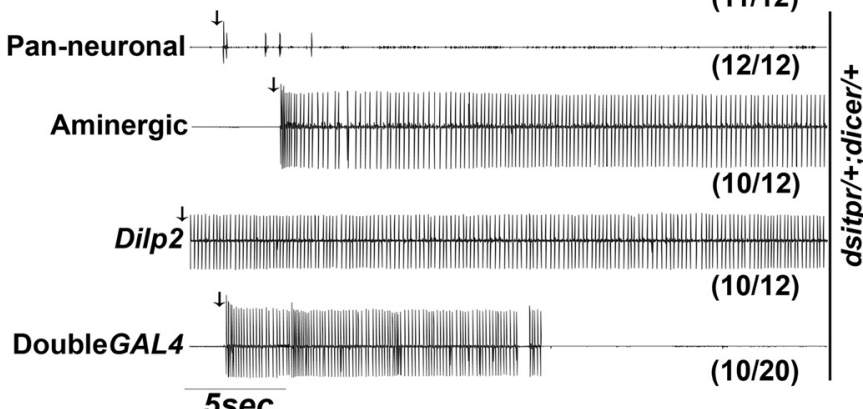

E

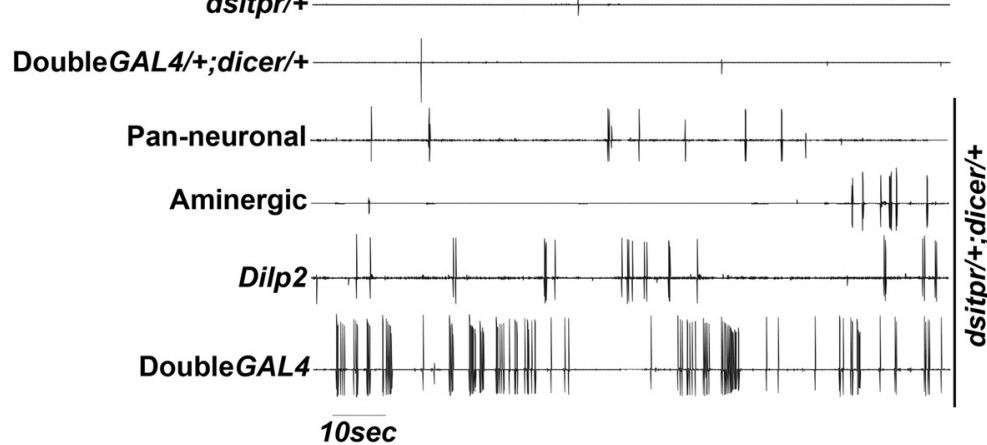

G

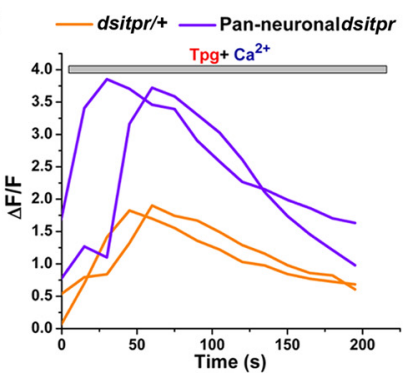

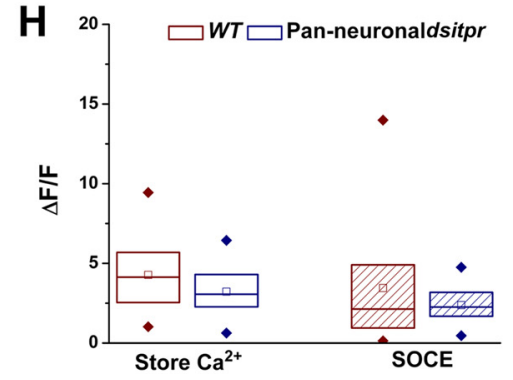

Figure 6. Reduced itpr levels in Drosophila neurons by dsRNA results in flight defects and lowered SOCE. $\boldsymbol{A}$, Pan-neuronal knockdown of itpr induces wing posture defects that are more pronounced in females and are further enhanced with a UASdicer (dicer) transgene in the background. Wing posture defects are not observed on dsitpr expression with DdcGAL4 (Aminergic) or Dilp2GAL4 (Dilp2) with a UASdicer transgene in the background. B, Significant flight defects are induced on pan-neuronal knockdown of itpr ( ${ }^{* *} p<0.005$; Student's $t$ test). C, Rhythmic action potentials in response to an air-puff stimulus are not observed on pan-neuronal knockdown of itpr. Simultaneous expression of $d s i t p r$ in DILP2 and aminergic domains (doubleGAL4) results in a reduced ability to maintain flight. $D$, Frequency of spontaneous firing in recordings from the DLMs is increased significantly on knockdown of itpr pan-neuronally or in the aminergic and DILP2 domains ( ${ }^{*} p<0.05$; Student's $t$ test). $\boldsymbol{E}$, Representative traces of spontaneously generated action potentials in recordings from the DLMs on pan-neuronal or tissue-specific knockdown of itpr ( $n \geq$ 12). $\boldsymbol{F}$, Pseudo-color image representation of measurement of store $\mathrm{Ca}^{2+}$ in neurons of the indicated genotypes by store depletion with thapsigargin followed by $\mathrm{Ca}^{2+}$ add-back to measure SOCE. Warmer colors represent higher $\mathrm{Ca}^{2+} . \mathbf{G}$, Single-cell traces of SOCE in individual cells of the indicated genotypes. Peak values of responses have been plotted as a box chart in (H). (Figure legend continues.) 
A

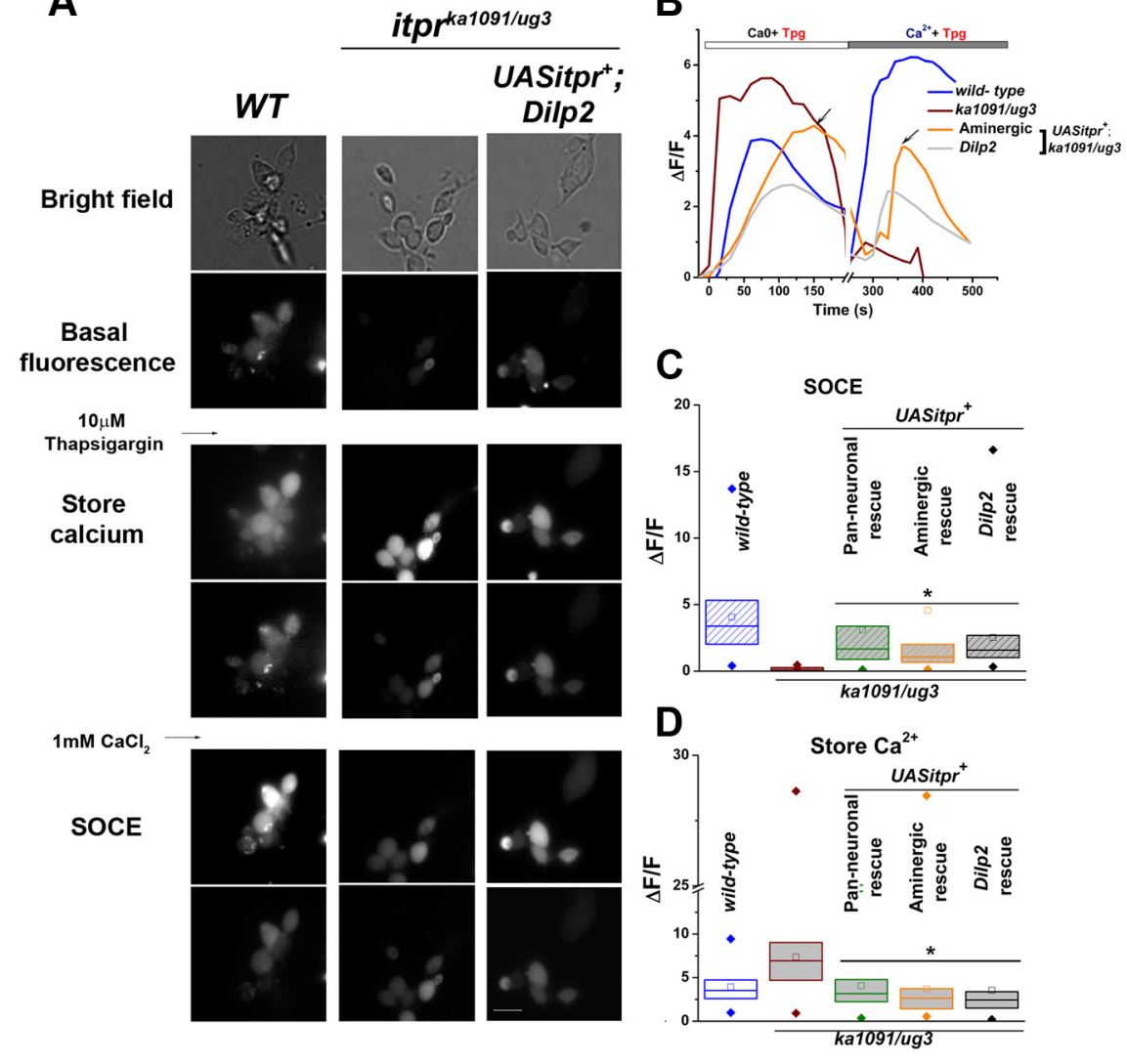

Figure 7. Restoration of store calcium and SOCE in itpr ${ }^{k a 1091 / u g 3}$ neurons upon targeted expression of itpr ${ }^{+}$to subneuronal domains. $A$, Fluorescent images show store $\mathrm{Ca}^{2+}$ in representative neurons of the indicated genotypes measured by store depletion with thapsigargin followed by $\mathrm{Ca}^{2+}$ add-back to measure SOCE. $\boldsymbol{B}$, Single cell traces of store $\mathrm{Ca}^{2+}$ and SOCE of the indicated genotypes. Arrowheads represent peak values of response which have been plotted as a box chart in $\boldsymbol{C}$ and $\boldsymbol{D}$. Targeted expression of $\mathrm{itpr}^{+}$to either aminergic or DILP2 neurons, in itpr ${ }^{\mathrm{ka1091/ug} 3}$ restores levels of store $\mathrm{Ca}^{2+}$ and SOCE in all the neurons. C, Box plot representation of the distribution of SOCE in neurons of the indicated genotypes. The bigger boxes represent the data spread as in Figure 6, smaller squares represent the mean and the diamonds on either side represent outlier upvalues. $\boldsymbol{D}$, Box plot comparison of store $\mathrm{Ca}^{2+}$ in larval neurons with pan-neuronal expression of $\mathrm{itpr}^{+}$ or those expressing itpr ${ }^{+}$specifically in the aminergic or DILP-producing cells. ${ }^{*} P_{\text {ANOVA }}<0.01$ compared with itpr ${ }^{\text {ka1091/ug3 } ;} ; P_{\text {ANOVA }}>$ 0.05 compared with wild-type, $n \geq 90$ cells).

can restore both flight and SOCE in itpr $r^{\text {ka1091/ug3 }}$ close to wildtype levels. The significance of elevated store $\mathrm{Ca}^{2+}$ in itpr ${ }^{\mathrm{kal0101/ug} 3}$ is not clear though it might potentially affect neuronal function (see also Venkiteswaran and Hasan, 2009). In itpr $r^{\text {wc703/ug3 }}$ store $\mathrm{Ca}^{2+}$ appears normal while loss-of-flight is equivalent to itpr ${ }^{\text {ka1091/ug } 3}$ suggesting that elevated store $\mathrm{Ca}^{2+}$ and flight deficits may not be related directly (data not shown).

In addition to normal SOCE, flight in itpr $r^{k a 1091 / u g 3}$ depends upon the extent of $\mathrm{Ca}^{2+}$ release through the $\mathrm{InsP}_{3} \mathrm{R}$ (Venkiteswaran and Hasan, 2009). This parameter of intracellular $\mathrm{Ca}^{2+}$ signaling was measured by pan-neuronal expression of $d S T I M^{+}$and $\mathrm{dOrai}^{+}$in neurons and simultaneous ectopic expression of the Drosophila muscarinic acetylcholine receptor (mAChR). The Drosophila $\mathrm{mAChR}$ responds to carbamylcholine (carbachol) with generation of $\mathrm{InsP}_{3}$ and $\mathrm{Ca}^{2+}$-release through the $\mathrm{InsP}_{3} \mathrm{R}$

(Figure legend continued.) H, Pan-neuronal expression of dsditpr lowers SOCE. Box plots representing the distribution of store $\mathrm{Ca}^{2+}$ and SOCE in neurons from the indicated genotypes. The bigger boxes represent the data spread (from 25 to $75 \%$ ), the horizontal line in each box is the median, smaller squares represent mean and the diamonds on either side represent outlier values ( ${ }^{*} P_{\text {Anova }}<0.05$ compared with dsitpr controls; $n \geq 150$ cells).
(Millar et al., 1995). Using this assay it has been demonstrated that $\mathrm{Ca}^{2+}$-release is significantly attenuated through mutant itpr $r^{\text {ka1091/ug } 3}$ channels (Venkiteswaran and Hasan, 2009). Pan-neuronal expression of either $\mathrm{dSTIM}^{+}$or $\mathrm{dOrai}^{+}$in the background of $i$ tpr ${ }^{k a 1091 / u g 3}$ led to higher $\mathrm{Ca}^{2+}$ release on stimulation with two different concentrations of carbachol (Fig. $8 D-G$; $\left.P_{\text {ANOVA }}<0.05\right)$. Direct measurement of $\mathrm{Ca}^{2+}$-release through the $\operatorname{InsP}_{3} \mathrm{R}$ in DILP2 and Ddc neurons is technically not feasible at present. However, based on these observations it is likely that expression of $d_{S T I M}{ }^{+}$in DILP2 and aminergic neurons of $i t r^{k a 1091 / u g 3}$ has a stimulatory effect on $\mathrm{InsP}_{3}$-mediated $\mathrm{Ca}^{2+}$ release. The mechanism of how STIM/Orai stimulates $\mathrm{Ca}^{2+}$-release through mutant Ins $\mathrm{P}_{3} \mathrm{Rs}$ is under investigation.

\section{Discussion}

In this study, we show that defective flight and associated physiology of itpr mutants can be rescued by expression of either $i t p r^{+}$or SSTIM $^{+}$in DILP2 neurons as well as in nonoverlapping aminergic neurons. This rescue of systemic phenotypes is accompanied by pan-neuronal restoration of intracellular calcium homeostasis, specifically SOCE, to wild-type levels. Moreover, while pan-neuronal knock-down of the Ins $\mathrm{P}_{3} \mathrm{R}$ mirrors itpr mutant phenotypes, knock-down of $i t p r^{+}, d S T I M^{+}$or $d \mathrm{Orai}^{+}$by tissue-specific dsRNA expression has a minimal effect on flight ability. These results support an important role for InsP $\mathrm{P}_{3} \mathrm{R}$ activity and SOCE in the function of DILP2 neurons and suggest that DILP2 and aminergic neurons can modulate neuronal function at systemic and cellular levels by non-cell-autonomous mechanisms.

Intracellular calcium homeostasis in DILP2 neurons and the control of flight circuit development

The rescue of flight in itpr mutants by itpr $r^{+}$expression in DILP2 neurons during pupal development suggests a role for Drosophila DILPs in development of the flight circuit. Several studies in both invertebrates and vertebrates have demonstrated that insulin participates in the neuronal remodeling of developing circuits. Motoneurons that innervate the indirect flight muscles in Drosophila undergo dendritic and axonal remodeling during pupation with extensive dendritic outgrowth after $20 \mathrm{~h}$ APF till adult eclosion (Consoulas et al., 2002). DILP release from the DILP2 neurons may thus be modulated by intracellular $\mathrm{Ca}^{2+}$ signaling and function in the process of dendritic outgrowth during the pupal stages. In Drosophila, insulin signaling contributes to guidance of photoreceptor cell axons from the retina to the brain during development of the visual system (Song et al., 2003). Insulin transport into the CNS appears to increase in the neonatal period (Banks, 2004) in mammalian systems, suggesting that peripheral insulin levels influence the formation of circuits in the developing brain (Needleman and McAllister, 2008). 
Table 1. Percentage of cells that show store-operated $\mathrm{Ca}^{2+}$ entry in the genotypes indicated

\begin{tabular}{|c|c|c|c|c|c|c|c|}
\hline \multirow[b]{2}{*}{ wild-type } & \multirow[b]{2}{*}{$i t p r^{k u}$} & \multirow{2}{*}{$\begin{array}{l}\text { itpr }{ }^{k u} \text { Pan-neuronal } \\
\text { itpr }^{+}\end{array}$} & \multirow{2}{*}{$\begin{array}{l}i t p r^{k u} \text { Aminergic } \\
\text { itpr }^{+}\end{array}$} & \multicolumn{4}{|c|}{ itpr ${ }^{k u}$ Dilp2GAL4 } \\
\hline & & & & $i t p r^{+}$ & $d S$ tim $^{+}$ & $\mathrm{dOrai}^{+}$ & $\mathrm{dStim}^{+} \mathrm{dOrai}^{+}$ \\
\hline 94.1 & 4.7 & 64.9 & 66.1 & 76.4 & 82.5 & 72 & 70.1 \\
\hline
\end{tabular}

Percentage values were obtained from the total number $(N \geq 90)$ of cells with measurable store $\mathrm{Ca}^{2+}$ upon thapsigargin addition.

Suppression of flight defects by expression of the SOCE regulator $\mathrm{ASTIM}^{+}$in DILP2 and aminergic neurons suggests that InsP $\mathrm{P}_{3} \mathrm{R}$-mediated $\mathrm{Ca}^{2+}$-release and SOCE cooperate to maintain intracellular $\mathrm{Ca}^{2+}$ homeostasis in these neurons, thus enabling $\mathrm{dSTIM}^{+}$to compensate for impaired $\mathrm{InsP}_{3} \mathrm{R}$ function. The partial compensation by $\mathrm{dOrai}^{+}$may be a consequence of reduced expression in the transgenic strain used. Alternately, STIM can also independently function in the regulation of basal and ER $\mathrm{Ca}^{2+}$ levels (Brandman et al., 2007) and this additional role may be relevant in improved maintenance of calcium homeostasis.

Despite rescue of adult itpr mutant phenotypes by aminergic and DILP2 neurons to similar extents, the two cellular domains exhibit no obvious overlap in either pupal or adult brains, indicating that the rescues are not mediated by a shared neuronal subset and suggests the existence of parallel modes of rescue. This is also supported by pan-neuronal knockdown of itpr, dSTIM or dOrai which results in wing posture and flight defects, but a similar effect is not seen on specific knockdown of these molecules in DILP2 or aminergic neurons. Thus perturbing intracellular calcium homeostasis in one neuronal domain is insufficient for inducing flight defects possibly due to compensation by other domains. The mildly enhanced phenotypes observed in the doubleGAL4 itpr knock-down support an interaction between the two expression domains. However, some of these results could also be a consequence of multiple modes of intracellular calcium regulation by alternate pathways in the DILP 2 and aminergic neurons. Our studies suggest that both aminergic and DILP2 neurons participate in development of the air-puff stimulated flight network in Drosophila. The existence of connections between serotonin and insulin signaling in Drosophila has been demonstrated as Drosophila Nucleostemin NS3 was found to act specifically within serotonergic neurons to regulate insulin signaling and exert global control over cell size and number (Kaplan et al., 2008). A similar mechanism might exist in the development and/or function of the flight circuit. However, a complete understanding of the individual neuronal components that constitute this flight network is currently lacking. Therefore, at this stage it is difficult to establish whether these domains physically constitute the flight circuit or indirectly influence its formation or function through secreted neuromodulators.

Similar systemic phenotypes are seen by knock-down of itpr, dOrai or ASTIM in the pan-neuronal domain (Venkiteswaran and Hasan, 2009) (Fig. 6). Pan-neuronal expression of wild-type itpr, dOrai and ASTIM however do not rescue itpr mutants to the same extent as rescue from the subneuronal domains, suggesting a developmental hierarchy for itpr mutant phenotypes beginning with wing posture. The rescue of individual phenotypes depends upon the level of pan-neuronal GAL4 expression at different stages of pupal development; normal wing posture is necessary but insufficient for restoration of flight physiology (Banerjee et al., 2004, 2006). Thus, unlike complete rescue, which requires GAL4 driven expression throughout flight circuit development, pan-neuronal knock-down at an early stage of pupal development produces wing posture defects, which abrogate normal flight. Data from the rescue of other adult neuronal phenotypes

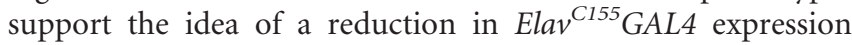

through pupal development (Zars et al., 2000) and a decrease in pan-neuronal GAL4 expression from larvae to adults is consistent with our observations of itpr rescue and RNAi knockdown.

\section{Correlating intracellular calcium homeostasis with flight behavior}

We show that SOCE and store $\mathrm{Ca}^{2+}$ are restored to wild-type levels in cultured larval neurons from itpr ${ }^{k a 1091 / u g 3}$ in conditions where flight and flight physiology are also rescued. Thus, expression of $\mathrm{dSTIM}^{+}$alone or $\mathrm{dSTIM}^{+}$and $\mathrm{dOrai}^{+}$simultaneously with Dilp2GAL4 suppresses flight and associated physiological defects while expression of $\mathrm{dOrai}^{+}$alone in the DILP neurons partially rescues wing posture and flight physiology but does not rescue flight ability and the maintenance of air-puff stimulated flight patterns (Venkiteswaran and Hasan, 2009). Interestingly, in neurons derived from Dilp2GAL4/UASdOrai ${ }^{+}$; itpr $^{\text {ka1091/ug3 }}$, SOCE remains significantly different from wild-type. Though correlative, these observations support the idea that wing posture defects, increased frequency of spontaneous firing and the inability to initiate flight can be suppressed by partial restoration of intracellular $\mathrm{Ca}^{2+}$ homeostasis in neurons. However, the maintenance of flight patterns and flight ability require normal SOCE in addition to the above parameters. The results obtained in this study parallel our previous observations, where a comprehensive rescue of flight and flight physiology is observed when $\mathrm{InsP}_{3}$ stimulated $\mathrm{Ca}^{2+}$-release and SOCE is brought back to wild-type levels in itpr $r^{\text {ka1091/ug3 }}$ (Venkiteswaran and Hasan, 2009).

A direct link between $\mathrm{InsP}_{3}$-mediated $\mathrm{Ca}^{2+}$ release and SOCE has been contested in vertebrates based primarily on data from DT40 cells in which all three InsP $\mathrm{P}_{3}$ Rs have been deleted (Broad et al., 2001; Ma et al., 2002). Interestingly, in Drosophila macrophages, SOCE through dOrai is coupled to intracellular $\mathrm{Ca}^{2+}$ release through the ryanodine receptor (RyR; Cuttell et al., 2008). Thus in Drosophila there appears to exist cell-type-specific coupling of either InsP $\mathrm{P}_{3} \mathrm{R}$ - or RyR-mediated $\mathrm{Ca}^{2+}$-release to SOCE. A similar dichotomy may exist among the kind of intracellular $\mathrm{Ca}^{2+}$-release channel used to trigger SOCE in different cell types in vertebrates. The extent and mechanism of activation of SOCE, by $\mathrm{InsP}_{3}$-mediated $\mathrm{Ca}^{2+}$ release, in Drosophila neurons needs further elucidation. These experiments will also help in understanding how dSTIM overexpression modulates mutant $\operatorname{Ins}_{3} \mathrm{R}$ function in cell-autonomous and non-cell-autonomous modes. At present these mechanisms can only be conjectured, since there does not appear to be a direct effect of itpr mutants and the rescued genotypes on dOrai and dSTIM levels (supplemental Fig. 4, available at www.jneurosci.org as supplemental material). Cell-autonomous rescue of mutant itpr function by dSTIM overexpression may be through the observed changes in luminal and/or cytosolic $\left[\mathrm{Ca}^{2+}\right]$. Non-cell-autonomous mechanisms are discussed below.

Non-cell-autonomous role of DILP2 neurons and aminergic neurons in regulating flight and calcium homeostasis $\mathrm{Ca}^{2+}$ measurements have been performed from a non-selective population of larval neurons in this study. The numbers of DILP or aminergic neurons in such conditions are a very small percent- 
A

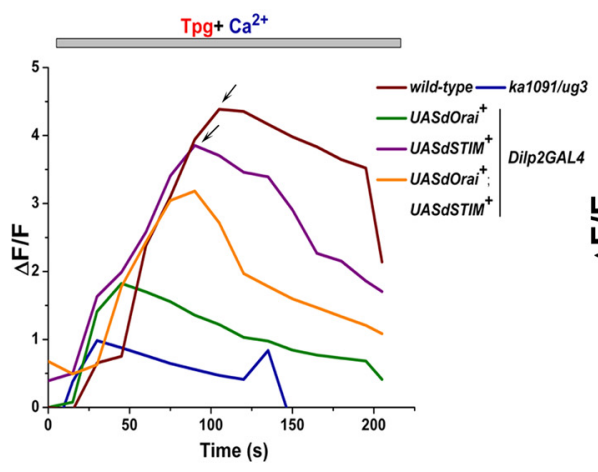

D

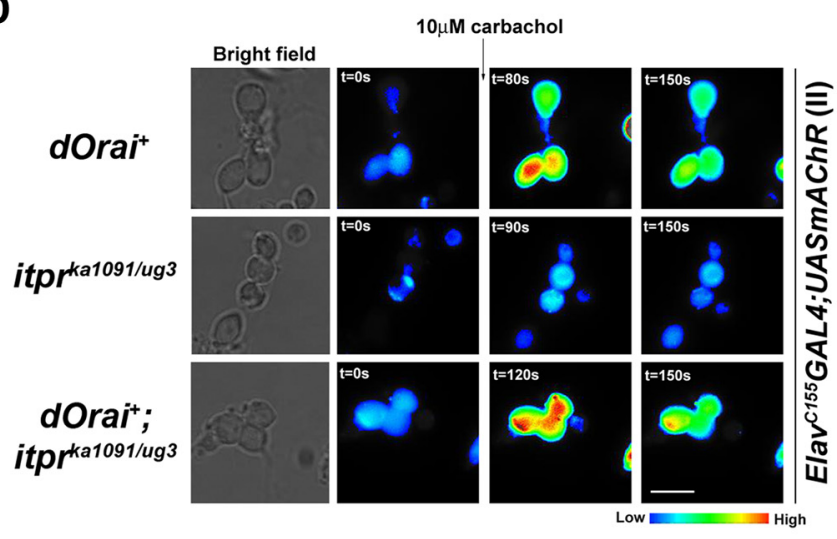

$\mathbf{F}$

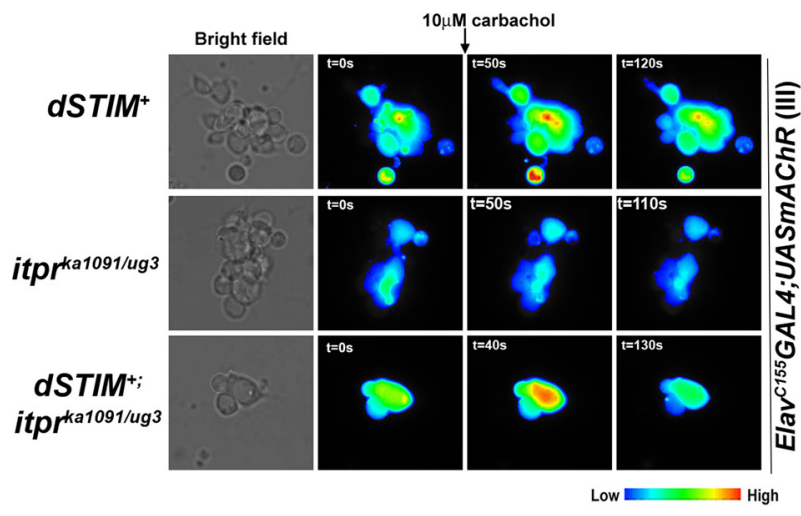

C
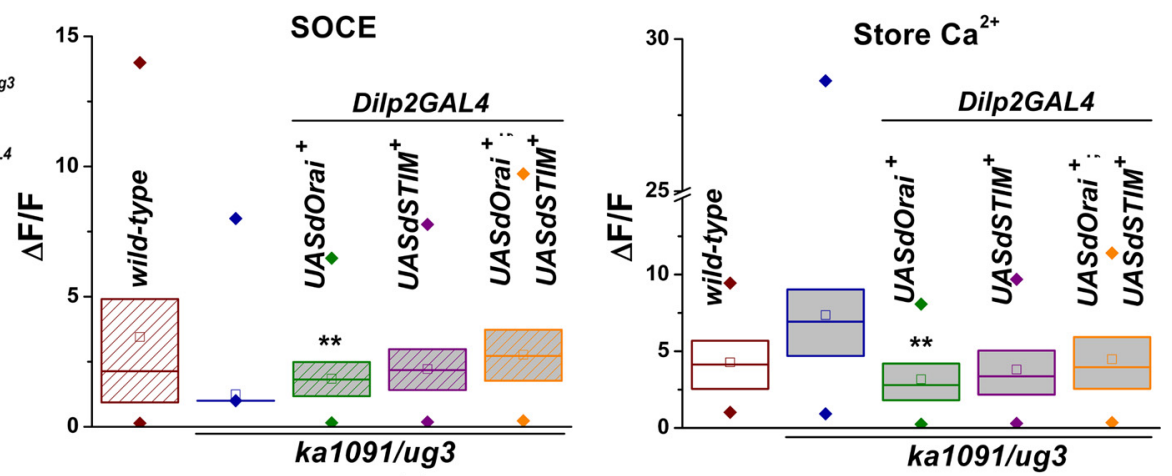

E

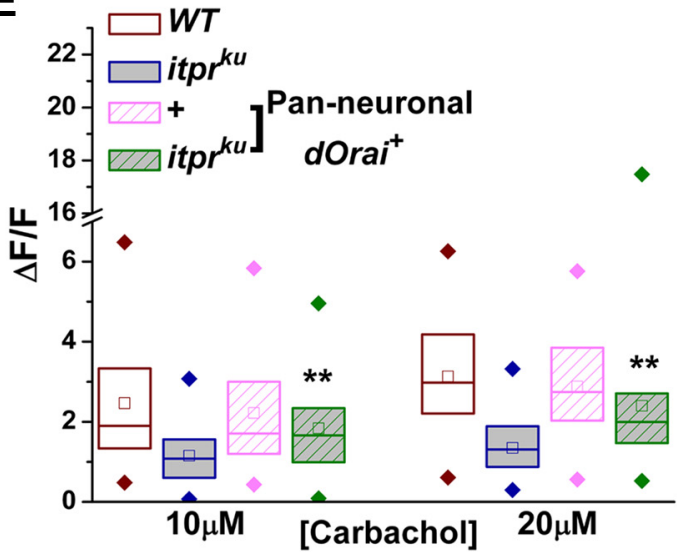

G

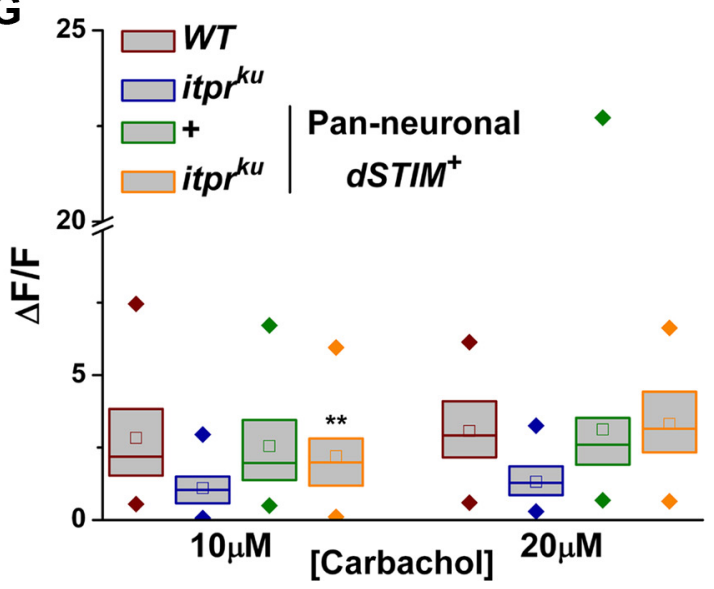

Figure 8. $\mathrm{dSTIM}^{+}$expression in itpr ${ }^{\mathrm{ka1091/ug3}}$ neurons restores intracellular $\mathrm{Ca}^{2+}$ homeostasis. $A$, Single cell traces of SOCE by $\mathrm{Ca}^{2+}$ add-back after store depletion. Arrowheads represent peak values of response which have been plotted as a box chart in $\boldsymbol{B}$. Box plots of $\triangle F / F$ values of SOCE $(\boldsymbol{B})$ and store $\mathrm{Ca}^{2+}(\boldsymbol{C})$ in the indicated genotypes. SOCE and store $\mathrm{Ca}^{2+}$ is significantly different

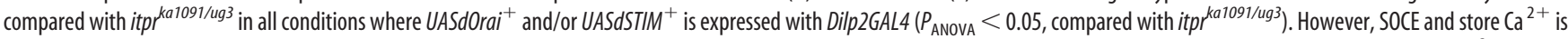
significantly different from wild-type only in the condition where UASdOrai ${ }^{+}$is expressed on its own with Dilp2GAL4 ( ${ }^{* *} P_{\text {ANOvA }}<0.05$, compared with wild-type). $D, F$, Magnitude of $C^{2+}{ }^{2+}$-release through mutant InsP $\mathrm{P}_{3}$ in itpr ${ }^{\mathrm{ka0197} / u g^{3}}$ neurons is significantly higher with pan-neuronal expression of either dOrai ${ }^{+}$or $\mathrm{dSTIM}^{+}$. Larval neurons of the indicated genotypes, with pan-neuronal expression of Drosophila $m A C h R$, were stimulated with $10 \mu \mathrm{m}$ carbachol and fluorescent images were taken in the time lapse mode. Images were pseudo-colored to represent $\left[\mathrm{Ca}^{2+}\right]_{\mathrm{i}}$. Warmer colors represent higher $\mathrm{Ca}^{2+} . \boldsymbol{E}, \mathbf{G}$, Box plots representing the magnitude of stimulated $\mathrm{Ca}^{2+}$ release in neurons of the indicated genotypes by 10 and $20 \mu \mathrm{m}$ carbachol. Expression of $d 0$ rai ${ }^{+}$partially restores stimulated release through $\operatorname{Ins}_{3} R$ such that it is significantly different from itpr ${ }^{k 1091 / u g 3}$, wild-type and controls $\left({ }^{* * P_{A N o v A}}<0.05\right)$. Expression of $d S T I M^{+}$restores stimulated release through Ins $P_{3} R$ such that it is significantly different from itpr $r_{10909 / 1 u g 3}\left(P_{\text {ANova }}<0.05\right)$ but not wild-type and controls $\left(P_{\text {ANovA }}>0.05\right)$ at $20 \mu \mathrm{m}$ carbachol. At $10 \mu \mathrm{m}$ carbachol, stimulated release on dSTIM ${ }^{+}$expression is significantly different from itpr ${ }^{k a 1091 / u g 3}$ and wild-type $\left({ }^{* *} P_{\text {ANovA }}<0.05\right)$ but not control (Elav ${ }^{\text {c155 }} /$ UASdSTIM $^{+} ;$UASmAChR, $\left.P_{\text {ANovA }}>0.05\right)$. In control cells expressing pan-neuronal dOrai ${ }^{+}$or $\mathrm{dSTIM}^{+}$expression (in the absence of itpr $\left.{ }^{\text {ka } 1091 / \mathrm{ug} 3}\right) \mathrm{Ca}^{2+}$ release through $\mathrm{InSP}_{3} \mathrm{R}$ is similar to wild-type $\left(P_{\mathrm{ANovA}}>0.05\right)$. Measurements from 150 cells or more were obtained for each box plot.

age of the total number of neurons analyzed (data not shown). Therefore, these results demonstrate that expression of $i t p r^{+}$or $\mathrm{dSTIM}^{+}$in a limited set of DILP2- or Ddc-expressing neurons restores calcium homeostasis in a wider number of unrelated neurons. As both these domains are predominantly neurosecre- tory and synthesize and release either insulin-like peptides or monoamines, it is likely that they can influence intracellular calcium homeostasis by secreted neuromodulators.

High levels of Drosophila insulin receptor $(d I R)$ mRNA are present in the larval and adult nervous system (Garofalo and 
Rosen, 1988) and dIR protein has been localized in the larval brain (Gorczyca et al., 1993). Drosophila serotonin receptors 5-HT1BDro (d5-HT1B) and 5-HT2Dro have been observed in both larval and adult brains (Yuan et al., 2005; Nichols, 2007). Therefore, a number of neurons could be targeted by secreted DILP or monoamines which might indirectly regulate the development and/or function of other neurons in the flight circuit.

Similar circuit outputs can be generated by multiple mechanisms (Marder and Goaillard, 2006). In the DdcGAL4 and Dilp2GAL4 rescue conditions the system may not be restored to a wild-type state at a cellular level but instead achieve new stable states in which a wild-type output is preserved (Greenspan, 2001). Our results demonstrate that for Drosophila flight, cellular calcium homeostasis in individual cells needs to be restored to a wild-type state and this then ensures the preservation of a normal physiological output. These observations are significant in the context of diseases where intracellular calcium signaling is deranged such as certain neurodegenerative and metabolic disorders (Verkhratsky, 2005; van de Leemput et al., 2007; Bezprozvanny and Mattson, 2008; Chan et al., 2009).

\section{References}

Agrawal N, Padmanabhan N, Hasan G (2009) Inositol 1,4,5-trisphosphate receptor function in Drosophila insulin producing cells. PLoS One 4:e6652.

Banerjee S, Hasan G (2005) The InsP3 receptor: its role in neuronal physiology and neurodegeneration. Bioessays 27:1035-1047.

Banerjee S, Lee J, Venkatesh K, Wu CF, Hasan G (2004) Loss of flight and associated neuronal rhythmicity in inositol 1,4,5-trisphosphate receptor mutants of Drosophila. J Neurosci 24:7869-7878.

Banerjee S, Joshi R, Venkiteswaran G, Agrawal N, Srikanth S, Alam F, Hasan G (2006) Compensation of inositol 1,4,5-trisphosphate receptor function by altering sarco-endoplasmic reticulum calcium ATPase activity in the Drosophila flight circuit. J Neurosci 26:8278-8288.

Banks WA (2004) The source of cerebral insulin. Eur J Pharmacol 490:5-12.

Benzer S (1973) Genetic dissection of behavior. Sci Am 229:24-37.

Berridge MJ (1998) Neuronal calcium signaling. Neuron 21:13-26.

Berridge MJ, Bootman MD, Roderick HL (2003) Calcium signalling: dynamics, homeostasis and remodelling. Nat Rev Mol Cell Biol 4:517-529.

Bezprozvanny I, Mattson MP (2008) Neuronal calcium mishandling and the pathogenesis of Alzheimer's disease. Trends Neurosci 31:454-463.

Borodinsky LN, Root CM, Cronin JA, Sann SB, Gu X, Spitzer NC (2004) Activity-dependent homeostatic specification of transmitter expression in embryonic neurons. Nature 429:523-530.

Brandman O, Liou J, Park WS, Meyer T (2007) STIM2 is a feedback regulator that stabilizes basal cytosolic and endoplasmic reticulum $\mathrm{Ca} 2+\mathrm{lev}-$ els. Cell 131:1327-1339.

Broad LM, Braun FJ, Lievremont JP, Bird GS, Kurosaki T, Putney JW Jr (2001) Role of the phospholipase C-inositol 1,4,5-trisphosphate pathway in calcium release-activated calcium current and capacitative calcium entry. J Biol Chem 276:15945-15952.

Cahalan MD (2009) STIMulating store-operated Ca(2+) entry. Nat Cell Biol 11:669-677.

Chan CS, Gertler TS, Surmeier DJ (2009) Calcium homeostasis, selective vulnerability and Parkinson's disease. Trends Neurosci 32:249-256.

Consoulas C, Restifo LL, Levine RB (2002) Dendritic remodeling and growth of motoneurons during metamorphosis of Drosophila melanogaster. J Neurosci 22:4906-4917.

Cuttell L, Vaughan A, Silva E, Escaron CJ, Lavine M, Van Goethem E, Eid JP, Quirin M, Franc NC (2008) Undertaker, a Drosophila Junctophilin, links Draper-mediated phagocytosis and calcium homeostasis. Cell 135:524-534.

Dietzl G, Chen D, Schnorrer F, Su KC, Barinova Y, Fellner M, Gasser B, Kinsey K, Oppel S, Scheiblauer S, Couto A, Marra V, Keleman K, Dickson BJ (2007) A genome-wide transgenic RNAi library for conditional gene inactivation in Drosophila. Nature 448:151-156.

Feske S, Gwack Y, Prakriya M, Srikanth S, Puppel SH, Tanasa B, Hogan PG, Lewis RS, Daly M, Rao A (2006) A mutation in Orail causes immune deficiency by abrogating CRAC channel function. Nature 441:179-185.
Friggi-Grelin F, Coulom H, Meller M, Gomez D, Hirsh J, Birman S (2003) Targeted gene expression in Drosophila dopaminergic cells using regulatory sequences from tyrosine hydroxylase. J Neurobiol 54:618-627.

Garofalo RS, Rosen OM (1988) Tissue localization of Drosophila melanogaster insulin receptor transcripts during development. Mol Cell Biol 8:1638-1647.

Gorczyca M, Augart C, Budnik V (1993) Insulin-like receptor and insulinlike peptide are localized at neuromuscular junctions in Drosophila. J Neurosci 13:3692-3704.

Greenspan RJ (2001) The flexible genome. Nat Rev Genet 2:383-387.

Hewavitharana T, Deng X, Soboloff J, Gill DL (2007) Role of STIM and Orai proteins in the store-operated calcium signaling pathway. Cell Calcium 42:173-182.

Hewes RS, Park D, Gauthier SA, Schaefer AM, Taghert PH (2003) The bHLH protein Dimmed controls neuroendocrine cell differentiation in Drosophila. Development 130:1771-1781.

Joshi R, Venkatesh K, Srinivas R, Nair S, Hasan G (2004) Genetic dissection of itpr gene function reveals a vital requirement in aminergic cells of Drosophila larvae. Genetics 166:225-236.

Kaplan DD, Zimmermann G, Suyama K, Meyer T, Scott MP (2008) A nucleostemin family GTPase, NS3, acts in serotonergic neurons to regulate insulin signaling and control body size. Genes Dev 22:1877-1893.

Kim SK, Rulifson EJ (2004) Conserved mechanisms of glucose sensing and regulation by Drosophila corpora cardiaca cells. Nature 431:316-320.

Li H, Chaney S, Roberts IJ, Forte M, Hirsh J (2000) Ectopic G-protein expression in dopamine and serotonin neurons blocks cocaine sensitization in Drosophila melanogaster. Curr Biol 10:211-214.

Liou J, Kim ML, Heo WD, Jones JT, Myers JW, Ferrell JE Jr, Meyer T (2005) STIM is a $\mathrm{Ca} 2+$ sensor essential for $\mathrm{Ca} 2+$-store-depletion-triggered Ca2 + influx. Curr Biol 15:1235-1241.

Lundell MJ, Hirsh J (1994) Temporal and spatial development of serotonin and dopamine neurons in the Drosophila CNS. Dev Biol 165:385-396.

Ma HT, Venkatachalam K, Parys JB, Gill DL (2002) Modification of storeoperated channel coupling and inositol trisphosphate receptor function by 2-aminoethoxydiphenyl borate in DT40 lymphocytes. J Biol Chem 277:6915-6922.

Marder E, Goaillard JM (2006) Variability, compensation and homeostasis in neuron and network function. Nat Rev Neurosci 7:563-574.

McGuire SE, Le PT, Osborn AJ, Matsumoto K, Davis RL (2003) Spatiotemporal rescue of memory dysfunction in Drosophila. Science 302:1765-1768.

McGuire SE, Roman G, Davis RL (2004) Gene expression systems in Drosophila: a synthesis of time and space. Trends Genet 20:384-391.

Mikoshiba K (2006) Inositol 1,4,5-trisphosphate IP(3) receptors and their role in neuronal cell function. J Neurochem 97:1627-1633.

Millar NS, Baylis HA, Reaper C, Bunting R, Mason WT, Sattelle DB (1995) Functional expression of a cloned Drosophila muscarinic acetylcholine receptor in a stable Drosophila cell line. J Exp Biol 198:1843-1850.

Needleman LA, McAllister AK (2008) Seeing the light: insulin receptors and the CNS. Neuron 58:653-655.

Nichols CD (2007) 5-HT2 receptors in Drosophila are expressed in the brain and modulate aspects of circadian behaviors. Dev Neurobiol 67:752-763.

Park JH, Schroeder AJ, Helfrich-Förster C, Jackson FR, Ewer J (2003) Targeted ablation of CCAP neuropeptide-containing neurons of Drosophila causes specific defects in execution and circadian timing of ecdysis behavior. Development 130:2645-2656.

Rulifson EJ, Kim SK, Nusse R (2002) Ablation of insulin-producing neurons in flies: growth and diabetic phenotypes. Science 296:1118-1120.

Soboloff J, Spassova MA, Tang XD, Hewavitharana T, Xu W, Gill DL (2006) Orail and STIM reconstitute store-operated calcium channel function. J Biol Chem 281:20661-20665.

Song J, Wu L, Chen Z, Kohanski RA, Pick L (2003) Axons guided by insulin receptor in Drosophila visual system. Science 300:502-505.

Spitzer NC (2002) Activity-dependent neuronal differentiation prior to synapse formation: the functions of calcium transients. J Physiol Paris 96:73-80.

Taghert PH, Hewes RS, Park JH, O’Brien MA, Han M, Peck ME (2001) Multiple amidated neuropeptides are required for normal circadian locomotor rhythms in Drosophila. J Neurosci 21:6673-6686.

van de Leemput J, Chandran J, Knight MA, Holtzclaw LA, Scholz S, Cookson MR, Houlden H, Gwinn-Hardy K, Fung HC, Lin X, Hernandez D, Simon-Sanchez J, Wood NW, Giunti P, Rafferty I, Hardy J, Storey E, 
Gardner RJ, Forrest SM, Fisher EM, et al. (2007) Deletion at ITPR1 underlies ataxia in mice and spinocerebellar ataxia 15 in humans. PLoS Genet 3:e108.

Venkatesh K, Siddhartha G, Joshi R, Patel S, Hasan G (2001) Interactions between the inositol 1,4,5-trisphosphate and cyclic AMP signaling pathways regulate larval molting in Drosophila. Genetics 158:309-318.

Venkiteswaran G, Hasan G (2009) Intracellular Ca2 + signaling and storeoperated $\mathrm{Ca} 2+$ entry are required in Drosophila neurons for flight. Proc Natl Acad Sci U S A 106:10326-10331.

Verkhratsky A (2005) Physiology and pathophysiology of the calcium store in the endoplasmic reticulum of neurons. Physiol Rev 85:201-279.

Vig M, Peinelt C, Beck A, Koomoa DL, Rabah D, Koblan-Huberson M, Kraft S, Turner H, Fleig A, Penner R, Kinet JP (2006) CRACM1 is a plasma membrane protein essential for store-operated $\mathrm{Ca} 2+$ entry. Science 312:1220-1223.
Wu CF, Suzuki N, Poo MM (1983) Dissociated neurons from normal and mutant Drosophila larval central nervous system in cell culture. J Neurosci 3:1888-1899.

Yuan Q, Lin F, Zheng X, Sehgal A (2005) Serotonin modulates circadian entrainment in Drosophila. Neuron 47:115-127.

Zars T, Fischer M, Schulz R, Heisenberg M (2000) Localization of a shortterm memory in Drosophila. Science 288:672-675.

Zhang SL, Yu Y, Roos J, Kozak JA, Deerinck TJ, Ellisman MH, Stauderman KA, Cahalan MD (2005) STIM1 is a Ca2 + sensor that activates CRAC channels and migrates from the $\mathrm{Ca} 2+$ store to the plasma membrane. Nature 437:902-905.

Zhang SL, Yeromin AV, Zhang XH, Yu Y, Safrina O, Penna A, Roos J, Stauderman KA, Cahalan MD (2006) Genome-wide RNAi screen of $\mathrm{Ca}(2+)$ influx identifies genes that regulate $\mathrm{Ca}(2+)$ release-activated $\mathrm{Ca}(2+)$ channel activity. Proc Natl Acad Sci U S A 103:9357-9362. 